\title{
Cardiac magnetic resonance imaging by retrospective gating: mathematical modelling and reconstruction algorithms
}

\author{
J. B. T. M. ROERDINK and M. ZWAAN \\ Centre for Mathematics and Computer Science, PO Box 4079, 1009 AB Amsterdam, The Netherlands
}

(Received in revised form 12 June 1992)

\begin{abstract}
This paper is concerned with some mathematical aspects of magnetic resonance imaging (MRI) of the beating human heart. In particular, we investigate the so-called retrospective gating technique which is a non-triggered technique for data acquisition and reconstruction of (approximately) periodically changing organs like the heart. We formulate the reconstruction problem as a moment problem in a Hilbert space and give the solution method. The stability of the solution is investigated and various error estimates are given. The reconstruction method consists of temporal interpolation followed by spatial Fourier inversion. Different choices for the Hilbert space $\mathscr{H}$ of interpolating functions are possible. In particular, we study the case where $\mathscr{H}$ is (i) the space of bandlimited functions, or (ii) the space of spline functions of odd degree. The theory is applied to reconstructions from synthetic data as well as real MRI data.
\end{abstract}

\section{Introduction}

Magnetic resonance imaging (MRI) is a diagnostic technique to measure and display crosssections of human organs. In this paper we consider the reconstruction of a cross-section of the beating heart. The general problem in dynamic MRI is that because of physical limitations, the standard measurement technique is not fast enough to acquire all the data necessary for the reconstruction of a single heart phase, in a time which is short enough that the motion of the heart is negligible. ${ }^{1}$

In the case of the beating heart, one can make use of the (approximate) periodicity of the motion. That is, data corresponding to the same relative heart phase may be recorded in different heartbeats. This presupposes exact reproducibility of the heart motion in successive cycles, a condition which will be violated in practice. There have been various ways to deal with this problem. McKinnon \& Bates [1], who considered cardiac imaging in the context of computerized tomography $(\mathrm{CT})$, assumed the number of cycles to be sufficiently small so that the heart motion during these cycles can be assumed to be 'quasistationary'.

Another alternative, which will be pursued in this paper, is to assume that there is a simple rule to map heart intervals of different duration to a standard heart interval of unit length in such a way that data are assigned to the correct heart phase. To perform this synchronization of the data, the electrocardiogram (ECG) is simultaneously recorded and

1 There are reports of attempts to do real time imaging (see e.g. Mansfield \& Morris [5]). 
used as a reference signal. The condition that the heart motion in different heart beats is identical up to a possible time rescaling will be referred to as the 'basic assumption' in the following. Since the synchronization of the data is carried out retrospectively, i.e. after data acquisition has been completed, the method has been referred to as 'retrospective gating' [2]; this is in contrast to ordinary gating or triggering, where the ECG is used for synchronization during data acquisition.

The organization of the paper is as follows. In $\S 2$ we first review the data acquisition process of MRI in general. Then we discuss the retrospective gating technique for cardiac imaging and explain how reconstructions at different heart phases can in principle be obtained. In $\S 3$ a mathematical problem formulation is given and we describe the previously obtained solution method [7-9]. We also consider optimal reconstruction methods in the presence of measurement noise. $\$ 4$ contains reconstructions from synthetic data as well from perturbed data. Reconstructions from real MRI data are presented in $\S 5$. In $\S 6$ we summarize the results, and present a discussion of future prospects.

\section{Cardiac magnetic resonance imaging}

In this section we give an introduction to dynamic cardiac imaging by MRI techniques. In particular, we explain the data acquisition based upon the concept of 'retrospective gating', as compared to the more conventional technique of ECG-triggered cardiac imaging. To make the paper reasonably self-contained, we start with a brief discussion of the principles of MRI for static objects. For background material on this topic the reader is referred elsewhere [3-6].

\subsection{Static MRI}

In a MR imaging system, the object is placed in a strong external magnetic field $\boldsymbol{B}$ along some axis, say the $z$-direction, ${ }^{2}$

$$
\boldsymbol{B}=\left(B_{0}+\boldsymbol{G} \cdot \boldsymbol{r}\right) \hat{z} .
$$

Here $B_{0}$ is a static homogeneous field and the $G \cdot \boldsymbol{r}$ term, with $\boldsymbol{G}=G_{x}(t) \hat{x}+G_{y}(t) \hat{y}+G_{z}(t) \hat{z}$, represents small field gradients whose strengths are controllable.

The basic purpose in MRI is to obtain an image of the density of protons in human tissue. Each proton has a 'spin', and can be considered as a small spinning top. When placed in a magnetic field $B_{0}$ each spin will perform a precession around the field direction with the so-called Larmor frequency $\omega_{L}=\gamma B_{0}$. Here $\gamma$ is a constant called the gyromagnetic ratio. The density of spins of the nuclear spin system is called the 'net magnetization' vector and denoted by $m$. After application of the main field $B_{0}$, the component $m_{z}$ of the magnetization in the $z$-direction (also referred to as the longitudinal magnetization) evolves towards thermodynamic equilibrium,

$$
m_{z}(t)=m_{\text {init }} \mathrm{e}^{-t / T_{1}}+m_{E Q}\left[1-\mathrm{e}^{-t / T_{1}}\right]
$$

where $T_{1}$ is the longitudinal (or spin-lattice) relaxation time, $m_{\mathrm{init}}$ is the initial magnetization and $m_{E Q}$ is the equilibrium magnetization.

\footnotetext{
$2 \hat{x}, \hat{y}$ and $\hat{z}$ denote unit vectors in the $x-, y$-and $z$-directions, respectively. Bold face symbols denote vectors.
} 
A spin system in equilibrium can be excited by applying a short magnetic field pulse $\boldsymbol{B}_{1}(t)$ at radiofrequency $(\mathrm{RF})$, with a frequency $\omega_{r f}$ which will usually be chosen equal to the Larmor frequency $\omega_{L}$ ('resonance condition'). After termination of the RF pulse, the spins start to get out of phase due to mutual interactions. This causes the transverse magnetization $m_{\perp}=m_{x}+i m_{y}\left(i^{2}=-1\right)$ to relax exponentially towards zero with a characteristic decay time $T_{2}$, called the transverse or spin-spin relaxation time:

$$
m_{\perp}(t)=m_{\perp}(0) \mathrm{e}^{-t / T_{2}} .
$$

At the same time, the longitudinal magnetization relaxes towards its equilibrium value $m_{E Q}$ with the relaxation time $T_{1}$, which is generally much larger than $T_{2}$. During relaxation, the excited spins give up energy at their Larmor frequencies in the form of radiofrequency radiation, which is recorded by a receiver $\mathrm{RF}$ coil. The resulting free induction decay (FID) signal is recorded in the form

$$
S(t)=\text { constant } \int m_{\perp}(\boldsymbol{r}, t) \mathrm{e}^{i\left(\omega_{L}-\omega_{r f}\right) t} \mathrm{~d} \boldsymbol{r} .
$$

\subsubsection{Spatial encoding of the MR signal}

To construct an image, the FID signal is spatially coded by making the magnetic field spatially varying so that the Larmor or resonance frequency becomes a function of position, e.g. by applying linear fields gradients (see (2.1)). If the system is again excited, now by a broad band RF pulse, the recorded signal has the form

$$
S(t)=\text { constant } \int m_{\perp}(\boldsymbol{r}, t) \exp \left[i\left(\int_{0}^{t} \mathrm{~d} t^{\prime} \omega\left(\boldsymbol{r}, t^{\prime}\right)-\omega_{r f} t\right)\right] \mathrm{d} \boldsymbol{r} .
$$

If we assume that the measuring time is much smaller than $T_{1}$ and $T_{2}$, and use the fact that the transverse magnetization $m_{\perp}(\boldsymbol{r}, 0)$ just after termination of the RF pulse is proportional to the local proton density $f(r)$, the recorded signal is approximately equal to

$$
S(t)=\text { constant } \int f(\boldsymbol{r}) \exp \left(-i \gamma \int_{0}^{t} \mathrm{~d} t^{\prime} \boldsymbol{G}\left(t^{\prime}\right) \cdot \boldsymbol{r}\right) \mathrm{d} \boldsymbol{r} .
$$

Writing $\xi=\left(\xi_{x}, \xi_{y}, \xi_{z}\right):=\gamma \int_{0}^{t} \mathrm{~d} t^{\prime} G\left(t^{\prime}\right)$, we recognize (2.6) as the Fourier coefficient of the function $f(r)$ at the Fourier frequency vector (or 'wave vector') $\xi$. By taking values of $S(t)$ at successive time intervals one obtains a so-called 'trajectory' in $\xi$-space, i.e. a sequence of samples of the Fourier transform of $f(\boldsymbol{r})$ [6]. For the later discussion of dynamic imaging we emphasize that it takes a finite amount of time to measure a trajectory.

Next we describe a method for choosing the gradient fields using square wave gradient pulses of constant magnitude and varying duration $[3,5]$. By turning on a $z$-gradient $G_{z}$ during excitation by the RF pulse, a very narrow slice perpendicular to the $z$-direction is excited. To provide spatial encoding within the selected plane, one subsequently turns on a phase-encoding gradient $G_{y}$ in the $y$-direction during a time $t_{y}$. This gives all the spins on the line $y=$ constant a phase factor $\gamma G_{y} t_{y}$. Finally, one applies a readout gradient $G_{x}$ in the $x$-direction, during which the FID signal is recorded and sampled every $\delta t$ seconds at times $t_{j}=\left(-\frac{1}{2} k_{x}^{\max }+j\right) \delta t, j=0,1, \ldots, k_{x}^{\max }-1$, where $k_{x}^{\max }$ (assumed to be even) is the number of 


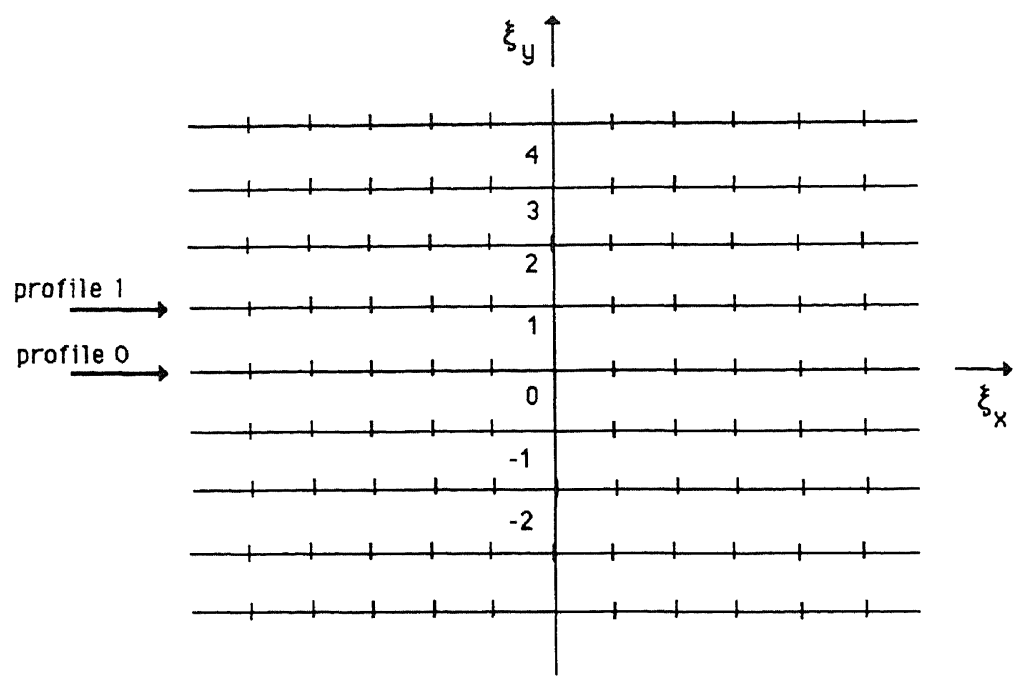

Figure 1. Profiles in the Fourier plane.

sampling points. Here the sampling time is set to zero in the middle of the measuring period. Similarly, $G_{y}$ is varied in steps of size $\Delta G_{y}$ from $-\frac{1}{2} k_{y}^{\max } \Delta G_{y}$ to $\left(\frac{1}{2} k_{y}^{\max }-1\right) \Delta G_{y}$ with $t_{y}$ fixed. The sequence of measurements $\left\{S\left(t_{j}\right): j=0, \ldots, k_{x}^{\max }-1\right\}$, with $G_{y}$ fixed, gives the Fourier transform for a discrete number of points on a horizontal line in the $\left(\xi_{x}, \xi_{y}\right)$-plane; such a horizontal trajectory is called a profile (see Fig. 1). A complete sequence of RF and gradient pulses is sketched in Fig. 2. To avoid aliasing by undersampling, both $\delta t$ and $\Delta G_{y}$ should be chosen in accordance with the Nyquist relation for sampling bandlimited functions:

$$
\Delta \xi_{x}=2 \pi / L_{x}, \quad \Delta \xi_{y}=2 \pi / L_{y}
$$

where $\Delta \xi_{x}$ and $\Delta \xi_{y}$ are the sampling distances in Fourier space and $L_{x}$ and $L_{y}$ are the fields of view in the $x$-and $y$-direction respectively. Since $\Delta \xi_{x}=\gamma G_{x} \delta t$ and $\Delta \xi_{y}=\gamma t_{y} \Delta G_{y}$ this yields

$$
\delta t=2 \pi /\left(\gamma G_{x} L_{x}\right), \quad \Delta G_{y}=2 \pi /\left(\gamma t_{y} L_{y}\right)
$$

Summarizing, the (normalized) sampled signal as a function of $\xi_{x}$ and $\xi_{y}$ equals

$$
S\left(\xi_{x}, \xi_{y}\right)=\int \mathrm{d} x \int \mathrm{d} y f(x, y) \mathrm{e}^{-i\left(\xi_{x} x+\xi_{y} y\right)},
$$

where $\xi_{x}=\gamma G_{x} \delta t k_{x}=\left(2 \pi / L_{x}\right) k_{x}$ and $\xi_{y}=\gamma t_{y} \Delta G_{y} k_{y}=\left(2 \pi / L_{y}\right) k_{y}$ with $k_{x}=-\frac{1}{2} k_{x}^{\max }, \ldots$, $\frac{1}{2} k_{x}^{\max }-1$ and $k_{y}=-k_{y}^{\max } / 2, \ldots, \frac{1}{2} k_{y}^{\max }-1$.

An estimate $\tilde{f}(x, y)$ of the proton density $f(x, y)$ of the selected slice is then constructed by performing the discrete inverse Fourier transform:

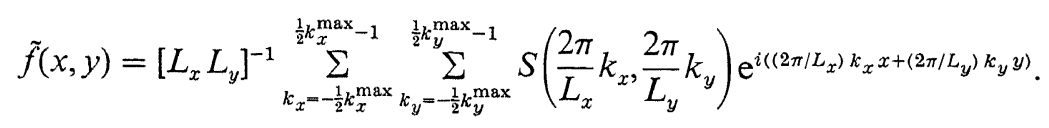

This transform can be efficiently implemented by the Fast Fourier Transform (FFT), see e.g. Nussbaumer [10]. 

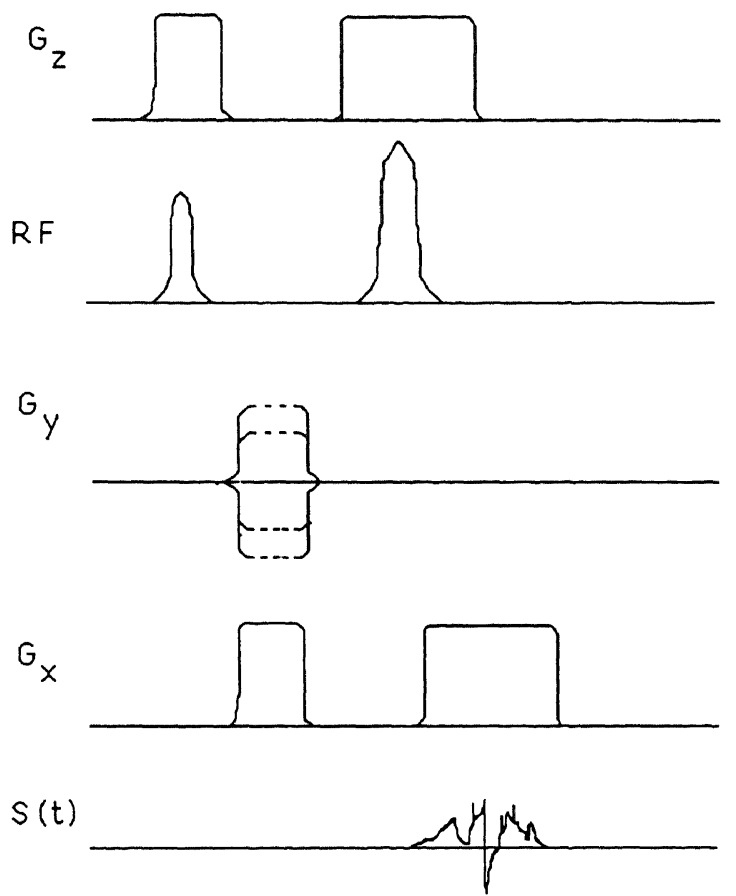

FIGURE 2. Sequences of gradient fields $G_{x}, G_{y}, G_{z}, r f$-pulse $R F$ and echo $S(t)$.

Apart from the various approximations made earlier (neglect of relaxation times, assumption of perfectly homogeneous fields, etc.), truncation of the sampling series is another cause of inaccuracy, leading to finite sampling errors such as ringing artefacts (Gibbs' phenomenon). These problems can be resolved by special signal processing techniques such as the use of data windows [11].

\subsection{Dynamic cardiac MRI}

\subsubsection{Acquisition principle of retrospective gating}

For diagnostic purposes a movie of the heart based upon several reconstructed heart phases will give useful information (e.g. cardiac output, heartwall motion, leaking heart valves) not easily obtained from static pictures. In the conventional ECG-triggered technique [12], the same slice is excited with a fixed number of RF pulses following the R-wave of the ECG. After this, the scanning system waits for the next R-wave before increasing the phaseencoding gradient. This means that no data are measured in the latter portion of relatively long heartbeats, leading to increased signal intensity during the first few frames of the image sequence, which manifests itself as a 'lightning' artifact.

The method of retrospective gating has been proposed to overcome these difficulties [2, $13,14]$. In this technique, there is an uninterrupted sequence of profile measurements with a fixed repetition time $T_{\mathrm{rep}}$. Recall that a profile is a sequence of measurements with $k_{y}$ fixed and $k_{x}$ increasing from $-\frac{1}{2} k_{x}^{\max }$ to $\frac{1}{2} k_{x}^{\max }-1$. Simultaneously, but independently of the 


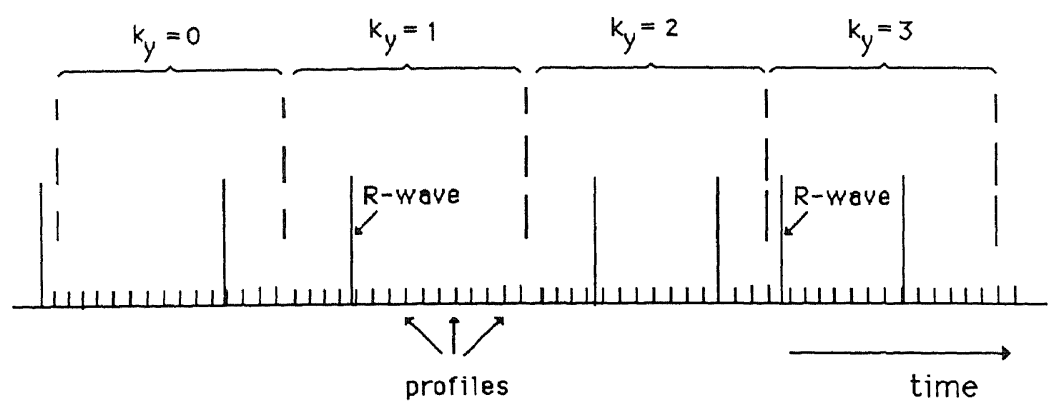

FIGURE 3. Acquisition method of retrospective gating. Large vertical bar: R-wave. Small vertical bar: a profile.

profile measurements, the ECG is recorded to enable a posteriori synchronization of the data to the correct heart phase (see Fig. 3). This means that the steady state condition of the spins is not disturbed, thus avoiding the lightning artefact. Another advantage is that the latter part of each heart cycle is covered too.

In principle, the value $k_{y}$ of the phase-encoding gradient should be increased after each heartbeat. But during the measuring process the information about the duration of the heartbeats is not available to control data acquisition. Therefore $k_{y}$ is increased after a fixed number $N_{\mathrm{pr}}$ of profile measurements. For example, one may take $N_{\mathrm{pr}}=\bar{T}_{\mathrm{RR}} / T_{\text {rep }}$, where $\bar{T}_{\mathrm{RR}}$ is the average RR-interval (time between two successive R-waves).

To get good image quality, the time resolution - as determined by the repetition time $T_{\text {rep }}$ - should be small enough. In practice, $T_{\text {rep }}$ may be of the order of $100 \mathrm{~ms}$ or less. To achieve this, a gradient sequence based on the so-called 'spin-echo technique' with a small flipangle $\theta$ is used, so that the spin system returns to equilibrium in a short time, after which the next profile can be measured.

To sum up, the data acquisition process contains the following steps:

(i) Initialize the phase encoding gradient: $k_{y}=-\frac{1}{2} k_{y}^{\max }$.

(ii) Measure profiles with a repetition time $T_{\text {rep }}$ until $N_{\text {pr }}$ profiles have been recorded. Each profile consists of $k_{x}^{\max }$ measurements $\left(k_{x}=-\frac{1}{2} k_{x}^{\max }, \ldots, \frac{1}{2} k_{x}^{\max }-1\right)$ of the Fourier transform of the cross-section, with $k_{y}$ fixed.

(iii) Increase the phase-encoding gradient: $k_{y} \rightarrow k_{y}+1$; go to (ii). If $k_{y}=\frac{1}{2} k_{y}^{\max }-1$, stop.

(iv) Simultaneously measure the times $R_{k}$ of occurrence of the R-waves.

\subsubsection{Reconstruction}

After data acquisition has been completed, we want to reconstruct pictures of the heart at various phases during the heartbeat. Because of the basic assumption (see the introduction), there exists a fixed RR-interval, referred to as the standard heart interval, on which to map the data recorded during heartbeats of different duration. Without loss of generality we take this fixed RR-interval as $I:=[0,1]$. For clarity, we will refer to original measurement time as 'time' $\tau$, and to relative time on the standard heart interval as 'phase' $t$. Probably the simplest rule to convert measurement time to heart phase is linear stretching, where the total duration of a heartbeat is used to renormalize the measurement times. 


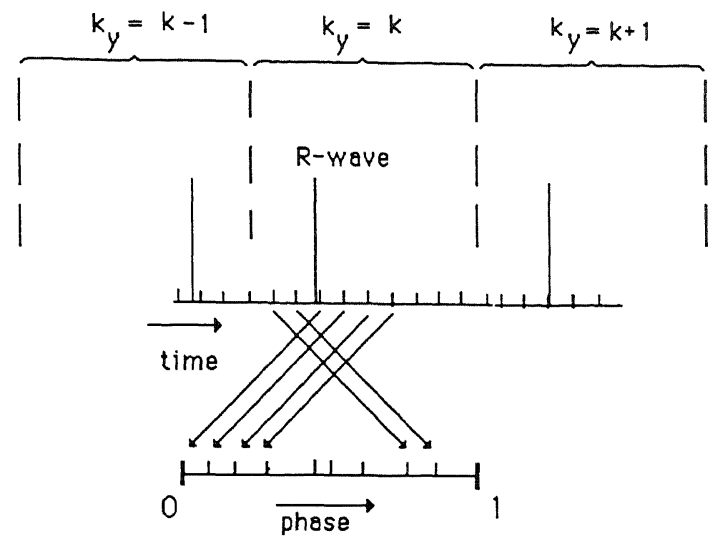

FIGURE 4. Reordering of the time markers by the time-to-phase conversion.

To make the above more precise we first introduce some notation. Let the proton density at position $(x, y)$ of a cross-section and at time $\tau$ be denoted by $F(x, y, \tau), \tau \in \mathbb{R}$. The function to be reconstructed is the proton density $f(x, y, t)$ at position $(x, y)$ and phase $t, 0 \leqslant t \leqslant 1$. So, if $D$ denotes a domain in the plane $\mathbb{R}^{2}$, we have that $F: D \times \mathbb{R} \rightarrow \mathbb{R}$ and $f: D \times I \rightarrow \mathbb{R}$. Each measurement of $F$ at time $\tau$ corresponds to a measurement of $f$ at a converted time $t(\tau)$ :

$$
F(x, y, \tau)=f(x, y, t(\tau)), \quad t \in[0,1] .
$$

In the case of linear stretching, the relation between $\tau$ and $t$ is

$$
t(\tau)=\frac{\tau-R_{k}}{R_{k+1}-R_{k}}, \quad \tau \in\left[R_{k}, R_{k+1}\right),
$$

where $R_{k}$ is the time at which the $k$ th $\mathrm{R}$-wave occurs, for $k=1,2, \ldots$.

Notice that as a result of the time-to-phase conversion, the data are reordered: when a new R-wave occurs while the value of $k_{y}$ is still unchanged, the next profile belongs to the beginning of the standard heart interval $I$, while the previous profile belongs to the end of $I$ (see Fig. 4). The next problem is that the reordered phases do not match with the wanted phases, which usually are a number of equally spaced values on the standard heart interval. Also, for each value of $k_{y}$ the pattern of reordered phases will be different. This problem can be solved by some form of interpolation. The details will be given in the next section.

In the acquisition method described above, the middle sample (corresponding to $k_{x}=0$ ) of the $i$ th profile at the $k_{y}$ th phase-encoding step occurs at time

$$
\tau_{i}\left(k_{y}\right)=\left(k_{y} N_{\mathrm{pr}}+i\right) T_{\text {rep }}, \quad i=0,1, \ldots, N_{\mathrm{pr}}-1,
$$

where $T_{\text {rep }}$ is the repetition time. Let the total measurement time of a single profile be denoted by $T_{\text {acq }}$. Then the time between two successive samples in a profile equals

$$
\delta t=T_{\mathrm{ac} \cdot \mathrm{q}} / k_{x}^{\max },
$$

since the total number of samples in a profile is $k_{x}^{\max }$. Therefore, the measurement of the $k_{x}$ th sample of the $i$ th profile at the $k_{y}$ th phase-encoding step takes place at the time

$$
\tau_{i}\left(k_{x}, k_{y}\right)=\tau_{i}\left(k_{y}\right)+k_{x} \delta t .
$$


At time $\tau_{i}\left(k_{x}, k_{y}\right)$ we measure the Fourier coefficient $\hat{F}\left(k_{x}, k_{y}, \tau_{i}\left(k_{x}, k_{y}\right)\right)$. After the time-tophase conversion, the Fourier coefficients of $F$ at the times $\tau_{i}\left(k_{x}, k_{y}\right)$ are interpreted as the Fourier coefficients of $f$ at the phases

$$
t_{i}\left(k_{x}, k_{y}\right):=t\left(\tau_{i}\left(k_{x}, k_{y}\right)\right)
$$

In the ideal case of a perfectly regular heartbeat (i.e. $R_{k+1}-R_{k}=\bar{T}_{R R}=N_{\mathrm{pr}} T_{\text {rep }}$ ) and in the case of linear stretching, one has

$$
t_{i}\left(k_{x}, k_{y}\right):=\frac{\tau_{i}\left(k_{x}, k_{y}\right)-R_{k}}{\bar{T}_{R R}}=\frac{i T_{\mathrm{rep}}+k_{x} \delta t}{\bar{T}_{R R}} .
$$

So in this case there is no dependence on $k_{y}$ and the sampling is uniform in $i$. However, in practice there will be both $k_{y}$-dependence and non-uniform sampling.

We thus end up with the following mathematical problem formulation:

Let $f: D \times I \rightarrow \mathbb{R}$ be a function $(x, y, t) \mapsto f(x, y, t)$, where $D$ is a rectangle of dimension $L_{x}$ by $L_{y}$ in the plane and $I=[0,1]$ a unit time interval. Let the spatial Fourier transform of $f$ be defined by

$$
\hat{f}\left(k_{x}, k_{y}, t\right):=\frac{1}{2 \pi} \iint_{D} f(x, y, t) \mathrm{e}^{-i\left(\left(2 \pi / L_{x}\right) k_{x} x+\left(2 \pi / L_{y}\right) k_{y} y\right)} \mathrm{d} x \mathrm{~d} y .
$$

Assume we have measured

$$
g_{i}\left(k_{x}, k_{y}\right):=\hat{f}\left(k_{x}, k_{y}, t_{i}\left(k_{x}, k_{y}\right)\right),
$$

for $k_{x}=-\frac{1}{2} k_{x}^{\max }, \ldots, \frac{1}{2} k_{x}^{\max }-1 ; k_{y}=-\frac{1}{2} k_{y}^{\max }, \ldots, \frac{1}{2} k_{y}^{\max }-1$ and $i=0, \ldots, N_{\mathrm{pr}}-1$. The problem then is: find a function $f: D \times I \rightarrow \mathbb{R}$ such that

$$
\hat{f}\left(k_{x}, k_{y}, t_{i}\left(k_{x}, k_{y}\right)\right)=g_{i}\left(k_{x}, k_{y}\right),
$$

for $k_{x}=-\frac{1}{2} k_{x}^{\max }, \ldots, \frac{1}{2} k_{x}^{\max }-1 ; k_{y}=-\frac{1}{2} k_{y}^{\max }, \ldots, \frac{1}{2} k_{y}^{\max }-1$ and $i=0, \ldots, N_{\mathrm{pr}}-1$.

To turn this question into a mathematically precise one, we have to specify the space of admissible solutions. This, together with the solution of the above problem, is the topic of the next section. Since the solution of this problem involves Fourier inversion in the spatial domain and interpolation in the time domain, we will refer to (2.20) as the mixed Fourierinterpolation problem, or simply, the mixed problem.

\section{Solution of the mixed Fourier-interpolation problem}

In this section we consider the mixed problem (2.20) which was formulated at the end of the previous section. To save writing we will use the notation $\boldsymbol{k}:=\left(k_{x}, k_{y}\right), g_{k, i}=g_{i}\left(k_{x}, k_{y}\right)$, $t_{k, i}=t_{i}\left(k_{x}, k_{y}\right), \mathbb{K}=\left\{\left(k_{x}, k_{y}\right): k_{x}=-\frac{1}{2} k_{x}^{\max }, \ldots, \frac{1}{2} k_{x}^{\max }-1 ; k_{y}=-\frac{1}{2} k_{y}^{\max }, \ldots, \frac{1}{2} k_{y}^{\max }-1\right\}$ and $\mathrm{0}=$ $\left\{i: i=0, \ldots, N_{\mathrm{pr}}-1\right\}$. Then the problem to be solved reads as follows:

Problem formulation Given a sequence of real numbers $\left\{t_{k, i}\right\}$ and a sequence of complex numbers $\left\{g_{k, i}\right\}$, find a function $f: D \times I \rightarrow \mathbb{R}$ such that

$$
\hat{f}\left(k, t_{k, i}\right)=g_{k, i}, \quad k \in K, i \in \mathbb{Q} .
$$


We assume that for each $\boldsymbol{k}$ the sequence $\left\{t_{i}(\boldsymbol{k})\right\}_{i \in \mathbb{1}}$ consists of distinct real numbers. This problem has been extensively studied by one of us $[7-9,15]$, and we briefly summarize the results here. For the details the reader is referred to the quoted references. First we consider the solution of problem (3.1) in a Hilbert space setting in $\S 3.1 . \S 3.2$ is concerned with stability and error estimates. Regularization is treated in $\S 3.3$ and in $\S 3.4$ we consider optimal reconstruction in the presence of noise.

\subsection{Moment problems in a Hilbert space}

The interpolation problem (3.1) has a natural decomposition into a space-dependent and a time-dependent component. We assume that for each $r:=(x, y) \in D$, the function $f(r): t \rightarrow$ $f(\boldsymbol{r}, t)$ is an element of a certain Hilbert space $\mathscr{H}$. The inner product on $\mathscr{H}$ is denoted by angular brackets $\langle\cdot, \cdot\rangle_{\mathscr{H}}$. The function $f$ itself is assumed to be an element of a larger Hilbert space $\mathscr{W}$ with inner product

$$
\langle f, g\rangle_{\mathscr{H}}:=\int_{D}\langle f(r), g(r)\rangle_{\mathscr{H}} \mathrm{d} \boldsymbol{r} .
$$

Let $\left\{h_{i}\right\}_{i \in \mathbb{}}$ be an orthonormal basis for $\mathscr{H}$ and $\left\{e_{k}\right\}_{k \in \mathbb{K}}$ be the (orthonormal) Fourier basis for $L^{2}(D)$,

$$
e_{k}(\boldsymbol{r})=(2 \pi)^{-1} \mathrm{e}^{i \boldsymbol{k} \cdot \boldsymbol{r}},
$$

where without loss of generality we have taken $D=[-\pi, \pi]^{2}$. By construction, $\left\{e_{k} h_{i}\right\}_{k \in \mathbb{K}, i \in \mathbb{1}}$ is an orthonormal basis for $\mathscr{W}=L^{2}(D, \mathscr{H})$ and any $f \in \mathscr{W}$ has the representation

$$
f=\sum_{k \in \mathbb{K}, i \in \mathbb{I}}\left\langle f, e_{k} h_{i}\right\rangle_{\mathscr{W}} e_{k} h_{i} .
$$

The (generalized) Fourier transform $\hat{f}(\boldsymbol{k}): t \mapsto \hat{f}(\boldsymbol{k}, t)$ of $f$ is defined by

$$
\hat{f}(\boldsymbol{k}):=\int_{D} f(\boldsymbol{r}) \overline{e_{k}(\boldsymbol{r})} \mathrm{d} \boldsymbol{r} .
$$

Furthermore, we assume that $\mathscr{H}$ is a so-called Reproducing Kernel Hilbert Space (RKHS) [16]: for all $t \in \mathbb{R}$, there exists an element $Q_{t} \in \mathscr{H}$ such that point evaluations can be written as inner products:

$$
h(t)=\left\langle h, Q_{t}\right\rangle_{\mathscr{H}}, \quad \text { for each } h \in \mathscr{H} .
$$

The function $Q: \mathbb{R}^{2} \rightarrow \mathbb{C}$ with $Q(t, s):=Q_{t}(s)=\left\langle Q_{t}, Q_{s}\right\rangle_{\mathscr{H}}$ is called the reproducing kernel.

For the mixed problem we can perform a similar reformulation: define $\varphi_{k, i}(t)=Q\left(t_{k, i}, t\right)$, $k \in \mathbb{K}, i \in \mathbb{Q}$. Then

$$
\hat{f}\left(\boldsymbol{k}, t_{k, i}\right)=\left\langle\hat{f}(\boldsymbol{k}), \varphi_{\boldsymbol{k}, i}\right\rangle_{\mathscr{H}},
$$

where $\hat{f}(\boldsymbol{k})$ is defined in (3.5). Since, for arbitrary $h \in \mathscr{H},\left\langle f, e_{\boldsymbol{k}} h\right\rangle_{\mathscr{W}}=\langle\hat{f}(\boldsymbol{k}), h\rangle_{\mathscr{H}}$, (3.1) can be reformulated as a so-called 'moment problem'

$$
\left\langle f, e_{k} \varphi_{k, i}\right\rangle_{\mathscr{W}}=g_{k, i}, \quad \forall \boldsymbol{k} \in \mathbb{K}, \forall i \in \mathbb{Q} .
$$


In practice we only have a finite number of measurements, so $\mathbb{K}$ and $\mathbb{t}$ will be finite index sets. Therefore (3.7) will not have a unique solution. This problem is solved by considering the so-called minimum norm solution, i.e., the solution of (3.7) with smallest norm [16]. We will only consider Hilbert spaces such that, for all $k \in \mathbb{K}$, the system $\left\{\varphi_{k, i}\right\}_{i \in \mathbb{B}}$ is linearly independent in $\mathscr{H}$ as long as the sequence $\left\{t_{k, i}\right\}_{i \in \Perp}$ consists of distinct real numbers. Then the system $\left\{e_{k} \varphi_{\boldsymbol{k}, i}\right\}_{i \in \mathbb{k}, k \in \mathbb{K}}$ is a linearly independent system of vectors spanning a linear subspace of $\mathscr{W}$, denoted by $\mathscr{W}_{n}$. The solution of minimal norm to problem (3.7) is unique, lies in $\mathscr{W}_{n}$ and is given by

$$
f=\sum_{k \in \mathbb{K}} c_{k} e_{k}
$$

where $c_{k}$ is defined by

$$
c_{k}=\sum_{i \in \rrbracket} g_{k, i} \psi_{k, i}
$$

Here $\psi_{\boldsymbol{k}, i}$, which is orthogonal to $\varphi_{\boldsymbol{k}, j}, j \neq i$, is given by

$$
\psi_{k, i}=\sum_{j \in \mathbb{l}} \overline{\left(G^{-1}(\boldsymbol{k})\right)_{i j}} \varphi_{k, j}
$$

where, for each $\boldsymbol{k} \in \mathbb{K}, G(\boldsymbol{k})$ is the so-called Gram matrix, defined by

$$
(G(\boldsymbol{k}))_{i j}=\left\langle\varphi_{k, j}, \varphi_{k, i}\right\rangle_{\mathscr{H}}=Q\left(t_{k, j}, t_{k, i}\right), \quad \forall i, j \in \mathbb{Q} .
$$

So in fact the solution is obtained by finding, for each fixed frequency vector $\boldsymbol{k}$, a solution $c_{k}$ to the moment problem (3.1) and then taking the inverse Fourier transform:

$$
f(\boldsymbol{r}, t)=\sum_{\boldsymbol{k} \in \mathbb{K}} c_{\boldsymbol{k}}(t) e_{\boldsymbol{k}}(\boldsymbol{r})=\sum_{\boldsymbol{k} \in \mathbb{K}} e_{k}(\boldsymbol{r}) \sum_{i, j \in \mathbb{l}} g_{\boldsymbol{k}, i} \overline{\left(G^{-1}(\boldsymbol{k})\right)_{i j}} \varphi_{j}(\boldsymbol{k}, t) .
$$

\section{Example 3.1. Bandlimited functions}

Let for each $\boldsymbol{r} \in D$ the temporal Fourier transform of $t \rightarrow f(\boldsymbol{r}, t)$ be zero outside the interval $[-r, r]$; here $r$ is called the bandwidth. Then $\mathscr{H}$ is the Paley-Wiener space $\mathbb{P}_{r}$ of bandwidth $r$ defined by $\mathbb{P}_{r}:=\left\{f \in L^{2}(\mathbb{R}): \operatorname{supp} \hat{f} \subset[-r, r]\right\}$, where 'supp' denotes the support of a function. The reproducing kernel for a given bandwidth $r$ is given by

$$
Q(t, s)=\sqrt{ }(r / \pi) \operatorname{sinc}_{r}(s-t \pi / r) .
$$

Here $\operatorname{sinc}_{r}$ denotes the $\operatorname{sinc}$-function which is defined by

$$
\operatorname{sinc}_{r}(t):=\left\{\begin{array}{cc}
\frac{\sin (r t)}{r t} & t \neq 0, \\
1 & t=0 .
\end{array}\right.
$$

For the mixed problem we first have to determine the bandwidth $r_{k}$ for each $k \in \mathbb{K}$, which is not known in advance. In that case we choose as the bandwidth $r:=\sup \left\{r_{k}: k \in \mathbb{K}\right\}$ where

$$
r_{k}:=\inf \left\{\frac{\pi}{\left|t_{k, i}-t_{k, i+1}\right|}: i \in \mathbb{Q}\right\} .
$$




\section{Example 3.2. Spline functions}

Here we follow mainly $[16,17]$. The mesh $\Delta$ of the interval $[a, b]$ is a sequence of distinct real numbers $\left\{t_{1}, \ldots, t_{I}\right\}$ such that $a=t_{1}<t_{2}<\ldots<t_{I}=b$. The space $\mathscr{K}^{m}$ of spline functions of degree $m$ is defined by $\mathscr{K}^{m}:=\left\{f \in C^{m-1}[a, b] \mid f\right.$ is a polynomial of degree $m$ or less, on each mesh interval\}. The reproducing kernel in the space $\mathscr{K}^{2 n-1}$ of splines of odd degree is given by

$$
Q(t, s):=\sum_{k=0}^{n-1} \frac{(t-a)^{k}(s-a)^{k}}{(k !)^{2}}+\sum_{k=0}^{n-1}(-1)^{n+k+1} \frac{(t-a)^{2 n-k-1}(s-a)^{k}}{(2 n-k-1) ! k !}+\frac{(-1)^{n}}{(2 n-1) !}(t-s)_{+}^{2 n-1},
$$

where $t_{+}^{k}:=t^{k}$ for $t \geqslant 0$ and zero for $t<0$.

\subsection{Stability and error estimates}

In this section we give error bounds for the aliasing error, the amplitude error and the time jitter error for the mixed problem in the case of interpolation by sinc and spline functions; details can be found elsewhere $[8,9,15]$.

\subsubsection{The aliasing error}

Suppose the function $f$ we have measured (i.e. $\hat{f}\left(\boldsymbol{k}, t_{i}\right)=g_{\boldsymbol{k}, i}$, for all $\boldsymbol{k} \in \mathbb{K}$ and for all $i \in \mathbb{Q}$ ) does not lie in the function space $L^{2}(D, \mathscr{H})$. However, the approximation $\tilde{f}$, being a solution to (3.1), is an element of $L^{2}(D, \mathscr{H})$. This causes an error,

$$
E_{\mathrm{al}}^{\mathscr{H}}:=\int_{D}\|f(\boldsymbol{r}, \cdot)-\tilde{f}(\boldsymbol{r}, \cdot)\|_{\infty} \mathrm{d} \boldsymbol{r},
$$

which is called the aliasing error. Here the supremum norm is defined by

$$
\|f(\boldsymbol{r}, \cdot)\|_{\infty}:=\sup _{t \in \mathbb{R}}|f(\boldsymbol{r}, t)| .
$$

We give a bound for the aliasing error for the mixed problem in the case of sinc-and splineinterpolation.

If $\mathscr{H}=\mathbb{P}_{r}$, we only have an estimate for the case of equidistant sampling: $t_{i}(\boldsymbol{k})=i$ for all $\boldsymbol{k} \in \mathbb{R}$ and $i \in \mathbb{Q}$

$$
E_{\mathrm{al}}^{\mathrm{P}_{r}} \leqslant \int_{D} \int_{\mathbb{R} \backslash[-r, r]}|\hat{f}(\boldsymbol{r}, \omega)| \mathrm{d} \omega \mathrm{d} \boldsymbol{r} .
$$

Here the Fourier transform is taken with respect to time, as is indicated by the Fourier variable $\omega$.

The following estimate for spline functions holds for arbitrary distinct sampling points (now the Fourier transform is taken with respect to space),

$$
E_{\mathrm{al}}^{x^{2 n-1}} \leqslant C\left(\sup _{\boldsymbol{k} \in \mathbb{K}} \Delta_{\boldsymbol{k}}\right) \sum_{\boldsymbol{k} \in \mathbb{K}}\left\|\frac{\partial}{\partial t} \hat{\tilde{f}}(\boldsymbol{k}, \cdot)-\frac{\partial}{\partial t} \hat{f}(\boldsymbol{k}, \cdot)\right\|_{L^{2}[a, b]},
$$

where $C=2 \pi^{2}$ and the mesh-width is defined by $\Delta_{k}:=\sup _{i \in \mathbb{1}}\left|t_{k, i+1}-t_{k, i}\right|$. 


\subsubsection{The amplitude error}

Suppose the data $\left\{g_{k, i}\right\}$ are perturbed to $\left\{g_{k, i}^{\prime}\right\}$. The solution that corresponds to the perturbed problem is called $f^{\prime}$ and satisfies

$$
\hat{f}^{\prime}\left(\boldsymbol{k}, t_{k, i}\right)=g_{\boldsymbol{k}, i}^{\prime} \quad \forall \boldsymbol{k} \in \mathbb{K}, \forall i \in \mathbb{Q} .
$$

The amplitude error is defined by

$$
E_{\mathrm{amp}}^{\mathscr{H}}:=\left(\int_{D}\left\|f(\boldsymbol{r}, \cdot)-f^{\prime}(\boldsymbol{r}, \cdot)\right\|_{\infty}^{2} \mathrm{~d} \boldsymbol{r}\right)^{\frac{1}{2}} .
$$

Let $\mathscr{H}=\mathbb{P}_{r}$ or $\mathscr{H}=\mathscr{K}^{2 n-1}$. The following estimate holds:

$$
E_{\mathrm{amp}}^{\mathscr{H}} \leqslant \sup _{\boldsymbol{k} \in \mathbb{K}}\left\|G^{-1}(\boldsymbol{k})\right\|^{\frac{1}{2}} C_{\mathscr{H}}\left\|g-g^{\prime}\right\|_{\left.\ell^{2}(\mathbb{K} \times]\right)} .
$$

Here $C_{\mathscr{H}}=1$ if $\mathscr{H}=\mathbb{P}_{r}$, and, if $\mathscr{H}=\mathscr{K}^{2 n-1}$,

$$
C_{\mathscr{K}^{2 n-1}}=\sup \left\{\left(\sum_{k=0}^{n-1} \frac{(b-a)^{2 k}}{k !^{2}}\right)^{\frac{1}{2}}, \frac{(b-a)^{n-\frac{1}{2}}}{(n-1) ! \sqrt{ } 2 n-1}\right\} .
$$

\subsubsection{The time jitter error}

Suppose the time points $\left\{t_{i}(\boldsymbol{k})\right\}$ are perturbed to $\left\{\boldsymbol{t}_{i}^{\prime}(\boldsymbol{k})\right\}$. The solution that corresponds to the perturbed problem is again denoted by $f^{\prime}$, and satisfies

$$
\hat{f}^{\prime}\left(\boldsymbol{k}, t_{i}^{\prime}(\boldsymbol{k})\right)=g_{k, i} \quad \forall i \in \mathbb{0} .
$$

The time jitter error is defined by

$$
E_{\mathrm{tj}}^{\mathscr{H}}:=\left(\int_{D}\left\|f(\boldsymbol{r}, \cdot)-f^{\prime}(\boldsymbol{r}, \cdot)\right\|_{\infty}^{2} \mathrm{~d} \boldsymbol{r}\right)^{\frac{1}{2}} .
$$

Assume (for each $\boldsymbol{k} \in \mathbb{K}$ ) the time points $\left\{t_{i}(\boldsymbol{k})\right\}_{i \in \rrbracket}$ to be a sequence of distinct real numbers. Let the time points $t_{i}(\boldsymbol{k})$ be perturbed to $t_{i}^{\prime}(\boldsymbol{k})$, such that,

$$
\left|t_{i}(\boldsymbol{k})-t_{i}^{\prime}(\boldsymbol{k})\right| \leqslant \gamma, \quad \forall \boldsymbol{k} \in \mathbb{K}, \forall i \in \mathbb{Q} .
$$

Let $\left\{\varphi_{\boldsymbol{k}, i}^{\prime}\right\}$ be the system of vectors corresponding to the perturbed problem and $\left(G^{\prime}(\boldsymbol{k})\right)_{i j}:=$ $\left\langle\varphi_{k, j}^{\prime}, \varphi_{k, i}^{\prime}\right\rangle_{\mathscr{H}}$ the associated Gram matrix. Then the following estimates hold:

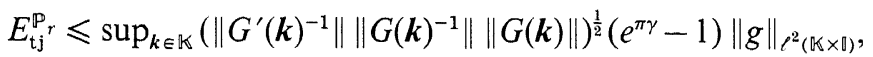

$$
\begin{aligned}
& E_{\mathrm{tj}}^{\mathscr{X}^{2 n-1}} \leqslant \sup _{k \in \mathbb{K}}\left(2\left\|G^{-1}(\boldsymbol{k})\right\|^{\frac{1}{2}}+\left\|G^{\prime^{-1}}(\boldsymbol{k})\right\|^{\frac{1}{2}}\left\|t_{\boldsymbol{k}}-t_{\boldsymbol{k}}^{\prime}\right\|_{\ell^{2}(0)}\right)\left(\sum_{\boldsymbol{k} \in \mathbb{K}}\left\|\frac{\partial}{\partial t} \hat{f}(\boldsymbol{k}, \cdot)\right\|_{\infty}^{2}+\left\|g_{\boldsymbol{k}}\right\|_{\ell^{2}(\theta)}^{2}\right)^{\frac{1}{2}} \text {. }
\end{aligned}
$$

\subsubsection{Conclusions about stability}

An inversion problem is called stable if small perturbations of the data yield small errors in the solution. From the estimates (3.20), (3.25) and (3.26) we conclude that problem (3.1) is stable for perturbation of the data and time points. We say that problem (3.1) is well- 
conditioned if the norms of the matrices are close to one. Otherwise it is called illconditioned. In the following we discuss the different error estimates.

The aliasing error for sinc-interpolation, formula (3.16), depends on the energy outside the band $[-r, r]$; if for each $\boldsymbol{r} \in D$, the measured function $f(r, \cdot)$ has a (temporal) Fourier transform with negligible energy outside $[-r, r]$, then the aliasing error will be small. The aliasing error for spline-interpolation, formula (3.17), depends on the temporal derivative. If the spline has a high order, then it tends to oscillate quickly. So, if the measured function does not oscillate quickly, the order of the interpolating splines should not be high.

The amplitude error for sinc- and spline-interpolation, formula (3.20), depends on the norm of $G(k)^{-1}$. If the time points $\left\{t_{k, i}\right\}_{i \in \mathbb{l}}$ (for a certain $k \in \mathbb{K}$ ) are lying close to each other, then the problem becomes ill-conditioned for perturbation of the data.

The time jitter error for sinc-interpolation, formula (3.25), becomes ill-conditioned for perturbation of the time points if (for a certain $k \in \mathbb{K}$ ) the elements of the sequences $\left\{t_{k, i}\right\}_{i \in \mathbb{I}}$ or $\left\{t_{k, i}^{\prime}\right\}_{i \in \mathbb{1}}$ are lying close to each other. The time-jitter error for spline-interpolation, formula (3.26), depends on $\left(G^{\prime}(\boldsymbol{k})\right)^{-1}$ and on the temporal derivative of the reconstructed function. So the problem becomes ill-conditioned for perturbation of the time points if (for a certain $k \in \mathbb{K})$ the elements of the sequence $\left\{t_{\boldsymbol{k}, i}\right\}_{i \in \mathbb{1}}$ are lying close to each other, or if the order of the spline is too high. This result will be illustrated by means of a simulation with synthetic images in $\S 4$.

\subsection{Regularization}

In those cases where the problem becomes ill-conditioned, we need to stabilize the solution methods. Procedures which deal with this problem go under the name of regularization [18]. Consider the following inversion problem. Let $T: \mathscr{H} \rightarrow \ell^{2}(\mathbb{0})$ be a bounded linear operator between the Hilbert spaces $\mathscr{H}$ and $\ell^{2}(\mathbb{Q})$. Let the data vector $g:=\left\{g_{i}\right\}_{i \in \mathbb{\emptyset}}$ be given. We want to find a function $f \in \mathscr{H}$ such that

$$
T f=g \text {. }
$$

If the inverse of $T$ exists and is bounded, then the problem can be solved directly in a stable manner. However, if this is not the case, then either the problem cannot be solved, or else the solution is not stable. To make up for this deficiency, we introduce a bounded linear operator $T^{\gamma}, \gamma>0$, from $\ell^{2}(\mathbb{D})$ into $\mathscr{H}$, such that $T^{\gamma} g$ minimizes the expression

$$
\|T f-g\|_{\ell^{2}}^{2}+\gamma\|f\|_{\mathscr{K}}^{2},
$$

over all $f \in \mathscr{H}$. The sequence $\left\{T^{\gamma}\right\}_{\gamma>0}$ is called the Tichonov-Phillips regularization of $T^{-1}$, and $\gamma$ is called the regularization parameter. It turns out (Natterer [19, p. 80]) that

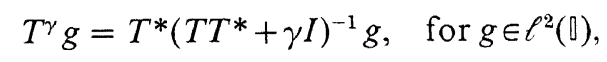

where $T^{*}$ denotes the adjoint operator of $T$ and $I$ is the identity operator on $\mathscr{H}$.

In the case of the mixed moment problem (3.7), one can derive from (3.28) (the proof is straightforward, but not trivial) that the corresponding Tichonov-Phillips regularization $\left\{\mathscr{T}^{\gamma}\right\}_{\gamma>0}$ is

$$
\left(\mathscr{T}^{\gamma} g\right)(\boldsymbol{r}, t)=\sum_{\boldsymbol{k} \in \mathbb{K}} e_{k}(\boldsymbol{r}) \sum_{i, j \in \mathbb{I}} g_{\boldsymbol{k}, i}\left((G(\boldsymbol{k})+\gamma I)^{-1}\right)_{i j} \varphi_{\boldsymbol{k}, j}(t),
$$

for $g:=\left\{g_{k, i}\right\}_{k \in \mathbb{K}, i \in \mathbb{I}} \in \ell^{2}(\mathbb{K} \times \mathbb{Q})$, and $G(\boldsymbol{k})$ the Gram matrix $(G(\boldsymbol{k}))_{i j}=\left\langle\varphi_{\boldsymbol{k}, j}, \varphi_{\boldsymbol{k}, i}\right\rangle_{\mathscr{H}}$. 
An important observation is that just as for the original problem, the regularized solution decomposes into a sum of solutions to smaller moment problems, one for each wave vector $\boldsymbol{k}$. For practical computer implementation, this has the advantage of reduced data storage requirements. Also, it opens up the possibility of parallel computation if speed is essential, as will be the case for medical applications.

\subsection{Optimal reconstruction in the presence of noise}

In the following we will consider a stochastic version of the moment problem (3.7) by adding external noise to the data. So consider the mixed problem

$$
\left\langle f, e_{k} \varphi_{k, i}\right\rangle_{\mathscr{W}}+u_{k, i}=g_{k, i}, \quad \forall \boldsymbol{k} \in \mathbb{K}, \forall i \in \mathbb{Q},
$$

where $\left\{u_{k, i}\right\}_{k \in \mathbb{K}, i \in \mathbb{\|}}$ is a sequence of random variables with zero mean and non-singular covariance matrix $V$ given by

$$
\mathbb{E}\left(u_{\boldsymbol{k}, i} u_{\boldsymbol{k}^{\prime}, j}\right)=V_{i j}\left(\boldsymbol{k}, \boldsymbol{k}^{\prime}\right)
$$

Here $\mathbb{E}$ denotes the mathematical expectation (statistical averaging) operator. The BLUE (Best Linear Unbiased Estimator) is given by [20-22] $\tilde{f}=T^{*}\left(T T^{*}+\gamma V\right)^{-1} g$. Under the assumption that the covariance matrix $V$ is diagonal in the Fourier variables, $V_{i j}\left(\boldsymbol{k}, \boldsymbol{k}^{\prime}\right)=$ $\delta_{k, k^{\prime}} Q_{i j}(k)$ for some non-singular matrix $Q$, one easily shows that

$$
\tilde{f}(\boldsymbol{r}, t)=\sum_{\boldsymbol{k} \in \mathbb{K}} e_{\boldsymbol{k}}(\boldsymbol{r}) \sum_{i, j \in \mathbb{I}} \varphi_{k, i}(t)\left[(G(\boldsymbol{k})+Q(\boldsymbol{k}))^{-1}\right]_{i, j} g_{\boldsymbol{k}, j},
$$

where $(G(\boldsymbol{k}))_{i, j}:=\left\langle\varphi_{\boldsymbol{k}, i}, \varphi_{\boldsymbol{k}, j}\right\rangle$ is the Gram matrix at a single wave vector $\boldsymbol{k}$ in the absence of noise. We thus recover the well known result that the optimal reconstruction in the presence of noise gives rise to a weighted Tichonov-Phillips regularization [23], which under the assumption of a diagonal covariance matrix decomposes again into a sum of interpolation problems for fixed $\boldsymbol{k}$, followed by Fourier inversion.

\section{Reconstruction of synthetic images}

In this section we describe the reconstruction of synthetic images, referred to as 'chest phantoms', which are a model of a cross-section of a beating human heart. We first describe the chest phantom $(\S 4.1)$ and the generation of the corresponding synthetic data ( $\$ 4.2)$. $\S 4.3$ discusses the reconstruction from synthetic data for various interpolation algorithms. Finally we consider reconstructions for perturbed synthetic data (§4.4).

\subsection{Definition of the chest phantom}

The chest phantom we use is taken as in [1], and is defined in terms of several (solid) ellipses, some of which are changing as a function of time (see Fig. 5).

The $k$ th ellipse $E_{k}$ is parametrized by five parameters $\alpha_{k}(t), \beta_{k}(t), \rho_{k}(t), \sigma_{k}(t)$ and $\theta_{k}(t)$, where the pair $\left(\alpha_{k}(t), \beta_{k}(t)\right)$ denotes the centre of the ellipse, $\rho_{k}(t)$ and $\sigma_{k}(t)$ are the length of the major and minor half axes and $\theta_{k}(t)$ is the angle of the ellipse with respect to the 


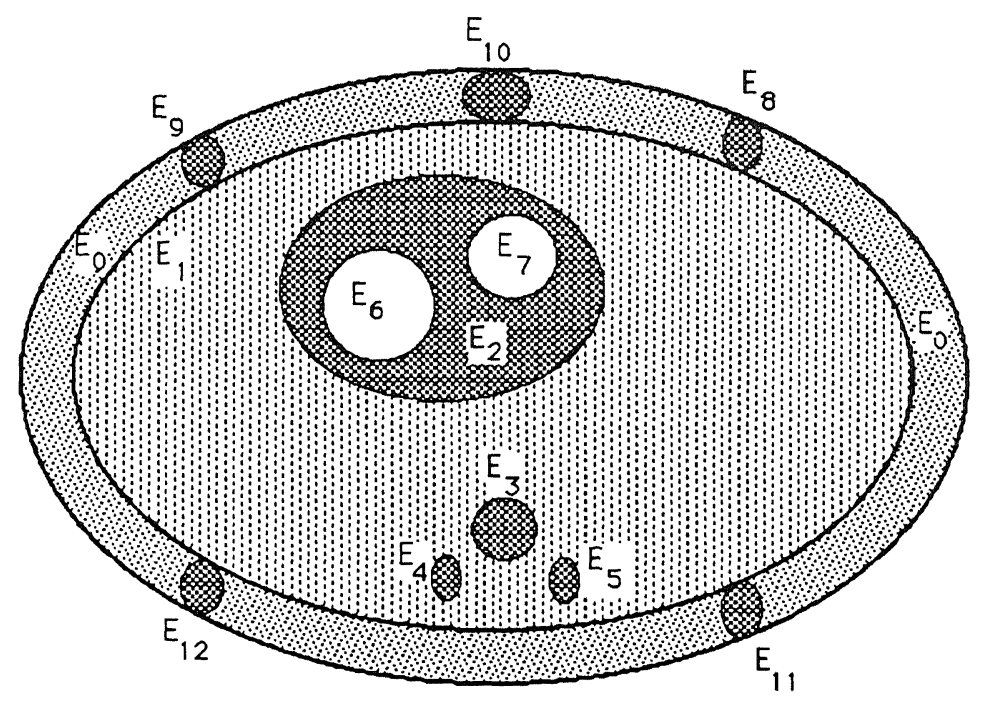

Figure 5. Sketch of the ellipses defining the chest phantom. Ellipses 2, 6 and 7 move periodically.

horizontal axis, all at time $t$. To every ellipse is assigned a 'grey-value'. The grey-value at a point equals the value associated to the smallest ellipse surrounding the point. The values of the parameters and grey-values of the ellipses are given in the following table, where $E_{0}$, $E_{1}$, etc., denotes the zeroth, first, etc. ellipse. The parameters $\alpha, \beta, \rho$ and $\sigma$ are given in pixel units, where we use a square of $256 \times 256$ pixels; $\theta$ is given in radians in units of $\pi / 16$ and grey-values range from 0 (white) to 255 (black). Here

$$
\begin{aligned}
& M_{21}(t)=35(1+0.3 \sin (2 \pi t+\pi / 4)), M_{22}(t)=28(1+0.3 \sin (2 \pi t+\pi / 4)) \\
& M_{61}(t)=112-8(1+0.3 \sin (2 \pi t+\pi / 4)+0.2 \sin (2 \pi t)) \\
& M_{62}(t)=105+11(1+0.3 \sin (2 \pi t+\pi / 4)+0.2 \sin (2 \pi t)) \\
& M_{63}(t)=M_{64}(t)=12(1+0.3 \sin (2 \pi t+\pi / 4)+0.2 \sin (2 \pi t)) \\
& M_{71}(t)=112+8(1+0.3 \sin (2 \pi t)+0.1 \sin (2 \pi t+\pi / 2)) \\
& M_{72}(t)=105-15(1+0.3 \sin (2 \pi t)+0.1 \sin (2 \pi t+\pi / 2)) \\
& M_{73}(t)=2 M_{74}(t)=10(1+0.3 \sin (2 \pi t)+0.1 \sin (2 \pi t+\pi / 2))
\end{aligned}
$$

So three of the ellipses carry out a periodic motion with period 1 generated by sinusoidal terms with the same frequency but different relative phases.

\subsection{Generation of synthetic data}

In the following we describe the simulation of the MRI measuring procedure in the case of our chest phantom (cf. §2.2). That is, we want to generate times $R_{k}$ of R-waves, measurement times $t_{k, i}$ and Fourier coefficients $g_{k, i}$ which will serve as input to the reconstruction procedures. 
Table 1 Parameters of the ellipses defining the chest phantom

\begin{tabular}{ccccccc}
\hline \hline & $\alpha$ & $\beta$ & $\rho$ & $\sigma$ & $\theta(\pi / 16$ rad $)$ & grey-value \\
\hline$E_{0}$ & 128 & 128 & 120 & 80 & 0 & 200 \\
$E_{1}$ & 128 & 128 & 110 & 70 & 0 & 128 \\
$E_{2}$ & 112 & 105 & $M_{21}(t)$ & $M_{22}(t)$ & 5 & 64 \\
$E_{3}$ & 128 & 175 & 10 & 16 & 0 & 64 \\
$E_{4}$ & 104 & 175 & 5 & 10 & -5 & 64 \\
$E_{5}$ & 152 & 175 & 5 & 10 & 5 & 64 \\
$E_{6}$ & $M_{61}(t)$ & $M_{62}(t)$ & $M_{63}(t)$ & $M_{64}(t)$ & 0 & 255 \\
$E_{7}$ & $M_{71}(t)$ & $M_{72}(t)$ & $M_{73}(t)$ & $M_{74}(t)$ & -5 & 255 \\
$E_{8}$ & 220 & 82 & 8 & 4 & -4 & 255 \\
$E_{9}$ & 36 & 82 & 8 & 4 & 4 & 255 \\
$E_{10}$ & 128 & 52 & 8 & 4 & 0 & 255 \\
$E_{11}$ & 220 & 174 & 8 & 4 & 4 & 255 \\
$E_{12}$ & 36 & 174 & 8 & 4 & -4 & 255 \\
\hline \hline
\end{tabular}

Generation of heart-interval times The times $R_{k}$ of $\mathrm{R}$-waves are sampled from a uniform distribution on the interval $\left[\bar{T}_{R R}(1-\epsilon), \bar{T}_{R R}(1+\epsilon)\right]$. Here $\epsilon$ is a parameter determining the relative variation of the heart-interval times. The case $\epsilon=0$ corresponds to a perfectly regular heartbeat.

Generation of measurement times This is free to the experimenter to choose. We will use the procedure of $\S 2.2$, formulae (2.13) and (2.15):

$$
\tau_{i}\left(k_{x}, k_{y}\right)=\left(k_{y} N_{p r}+i\right) T_{\mathrm{rep}}+k_{x} \delta t,
$$

where $\left(k_{x}, k_{y}\right) \in \mathbb{K}, i \in \mathbb{I}$, and where $N_{p r}$ is the number of profiles, $T_{\text {rep }}$ the repetition time, and $\delta t$ the time between successive samples in a profile.

Generation of Fourier coefficients Let a single heartbeat of the chest phantom be denoted by $f(\boldsymbol{r}, t)$, where $r \in D, t \in[0,1]$. We extend this function to a larger interval $\left[0, R_{J}\right) \subset \mathbb{R}$ by 'inverting' the time-to-phase conversion of $\S 2.2$. That is, given the sequence of times $R_{k}$ of R-waves, $0=R_{1}<R_{2}<R_{3}<\ldots<R_{J}$, we define $F: D \times \mathbb{R} \rightarrow \mathbb{B}$ by (cf. formula (2.12))

$$
F(\boldsymbol{r}, \tau):=f(\boldsymbol{r}, t(\tau)), \quad \text { for } \tau \in\left[R_{j}, R_{j+1}\right),
$$

where

$$
t(\tau)=\frac{\tau-R_{j}}{R_{j+1}-R_{j}} .
$$

That is, $F$ consists of a sequence of shifted and rescaled copies of $f$, modelling the beating human heart with varying $R R$-intervals. From the formula (4.2), the Fourier coefficients of this function $F$ with respect to the spatial parameters $\boldsymbol{r}=(x, y)$, are given by $\hat{F}\left(\boldsymbol{k}, \tau_{i}(\boldsymbol{k})\right)=$ $\hat{f}\left(\boldsymbol{k}, t\left(\tau_{i}(\boldsymbol{k})\right)\right)$. Since $f$ is the known synthetic image, the corresponding Fourier coefficient can be computed as soon as the rescaled times $t\left(\tau_{i}(\boldsymbol{k})\right)$ have been determined. This has to be done for all $\boldsymbol{k} \in \mathbb{K}, i \in \mathbb{\mathbb { V }}$. 


\subsection{Reconstruction from synthetic data}

In the following we compare the original (the phantom) with several reconstructions $f$. Input of the reconstruction algorithms (as described in $\S 3$ ) are the times $R_{k}$ of occurrence of the R-waves, the measurement times $\tau_{k, i}$ and Fourier coefficients $g_{k, i}=\hat{F}\left(\boldsymbol{k}, \tau_{k, i}\right)$ as described above. The reconstructions are performed by first interpolating in the timedomain for each fixed Fourier frequency $\boldsymbol{k}$, followed by Fourier inversion at a number $\Phi_{\max }$ of equidistant phases

$$
\phi_{i}=i / \Phi_{\max }, \quad i=0,1, \ldots, \Phi_{\max }-1 .
$$

For the time-interpolation we use first or third order spline interpolation and sinc interpolation, referred to as order 1 reconstruction, sinc reconstruction, etc. The interpolating functions in the case of order 1 and order 3 reconstruction are forced to be periodic by the use of appropriate boundary conditions. In the case of sinc-reconstruction this cannot be done, which causes the first and last phases of the reconstruction to be poorer in quality than the middle phases (see below). We also implemented the original technique of Bohning [14], referred to as order 0 reconstruction. Here the value at phase $\phi_{i}$ is taken to be the average of the data in the interval $\left[\phi_{i}, \phi_{i+1}\right)$, where a value zero is assigned if no data fall in this interval. If $\Phi_{\max }$ is chosen larger than $N_{\mathrm{pr}}$, this results in many assignments of a value zero to the interpolated Fourier coefficients, leading to severe aliasing artefacts (see e.g. Fig. 8).

The time markers $\tau_{i}\left(k_{x}, k_{y}\right)$ depend on both $k_{x}$ and $k_{y}$. For computational efficiency, we neglect the (small) measurement time interval $T_{\text {acq }}$ of a complete profile and assume that all data within one profile are measured at the same time, implying that the measurement times $t_{k, i}$ only depend on $k_{y}$. Since the Gram matrix $G(k)$ in formulae (3.29) or (3.32) depends on $\boldsymbol{k}$ through $t_{\boldsymbol{k}, i}$ (see $\S 3.1$ ), $G(\boldsymbol{k})$ also depends on $k_{y}$ only. So we only have to perform one, instead of $k_{x}^{\max }$, Gram matrix inversions per profile, resulting in a marked increase in computational efficiency. For the value of $T_{\text {acq }}=0.01$ used in the reconstructions below, we found that the neglect of $T_{\text {acq }}$ results in an error of at most $5 \%$. If, after rescaling, two time markers coincide the corresponding measurements are averaged. To prevent numerical instability we introduced in the case of order 3 and sinc interpolation a small number 'interval': time markers that lie within a distance 'interval' from each other are considered to be at the middle of this interval and the corresponding measurements are again averaged. This procedure can be considered as an ad hoc form of regularization. We also used regularized sinc-interpolation by the Tichonov-Phillips method (referred to as reg. sinc below) with a value $\gamma=0.01$ for the regularization parameter. (We also experimented with very large values of 'interval' in the (unregularized) sinc reconstruction: then the reconstruction errors become comparable to the results of reg. sinc, but the amount of spatial blurring in the region with moving ellipses becomes much larger.)

The reconstructed functions are computed at a rectangular pixel grid of dimensions $X_{\max }$ by $Y_{\max }$. The following parameter values were used in the generation of synthetic data:

$$
k_{x}^{\max }=k_{y}^{\max }=128, T_{\mathrm{acq}}=0.01, \epsilon=0.25 .
$$

The value of $\bar{T}_{R R}$ is arbitrarily taken to be unity (it is irrelevant because of the time-to-phase conversion), whereas the repetition time is determined by $T_{\text {rep }}=\bar{T}_{R R}(1+\epsilon) / N_{\text {pr }}$, so that also 

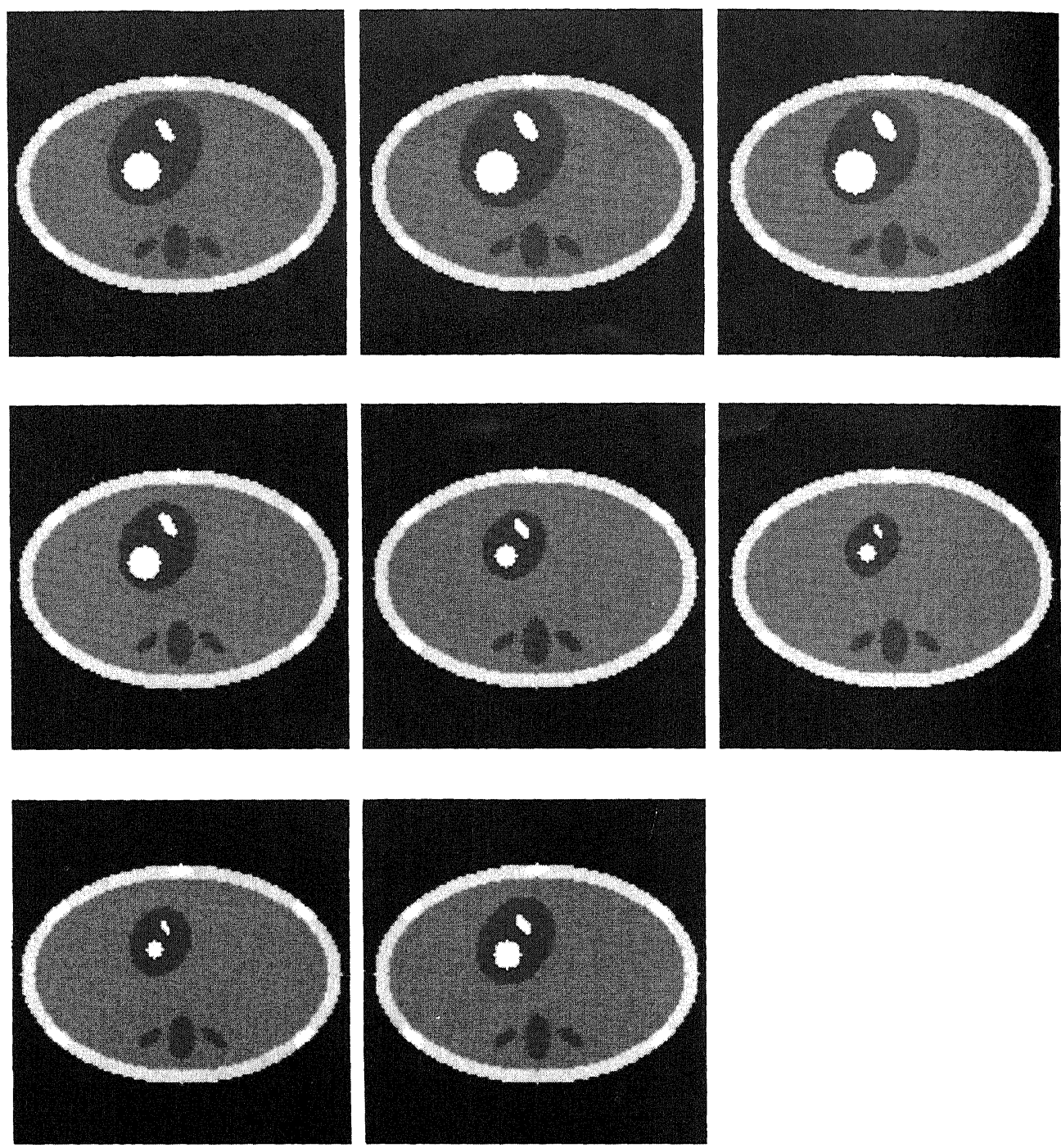

Figure 6. Original chest phantom. From left to right, top to bottom: phases 0-7.

in the longest heartbeat (which has length $\bar{T}_{R R}(1+\epsilon)$ ) the full number $N_{\mathrm{pr}}$ of profiles is measured. For the reconstructions we use the values

$$
X_{\max }=Y_{\max }=128, \Phi_{\max }=8 .
$$

The reconstructed phases are numbered from 0 to 7 . Original images are shown in Fig. 6. Reconstructions are presented for $N_{\mathrm{pr}}=15$ (Fig. 7) and $N_{\mathrm{pr}}=5$ (Fig. 8). In each case the first row displays order 0 reconstruction, the second row order 1 , the third one order 3 , the fourth one sinc-reconstruction and the fifth regularized sinc-reconstruction. In each row we show reconstructions at phases 0,3 and 6 from left to right. In the case that $N_{\mathrm{pr}}=5$ we see that the first and third order reconstructions are comparable in performance and give better results than zeroth order reconstruction. The (regularized) sinc-reconstruction is of lower quality, especially in the first phases, which may be due to the intrinsic nonperiodicity of 

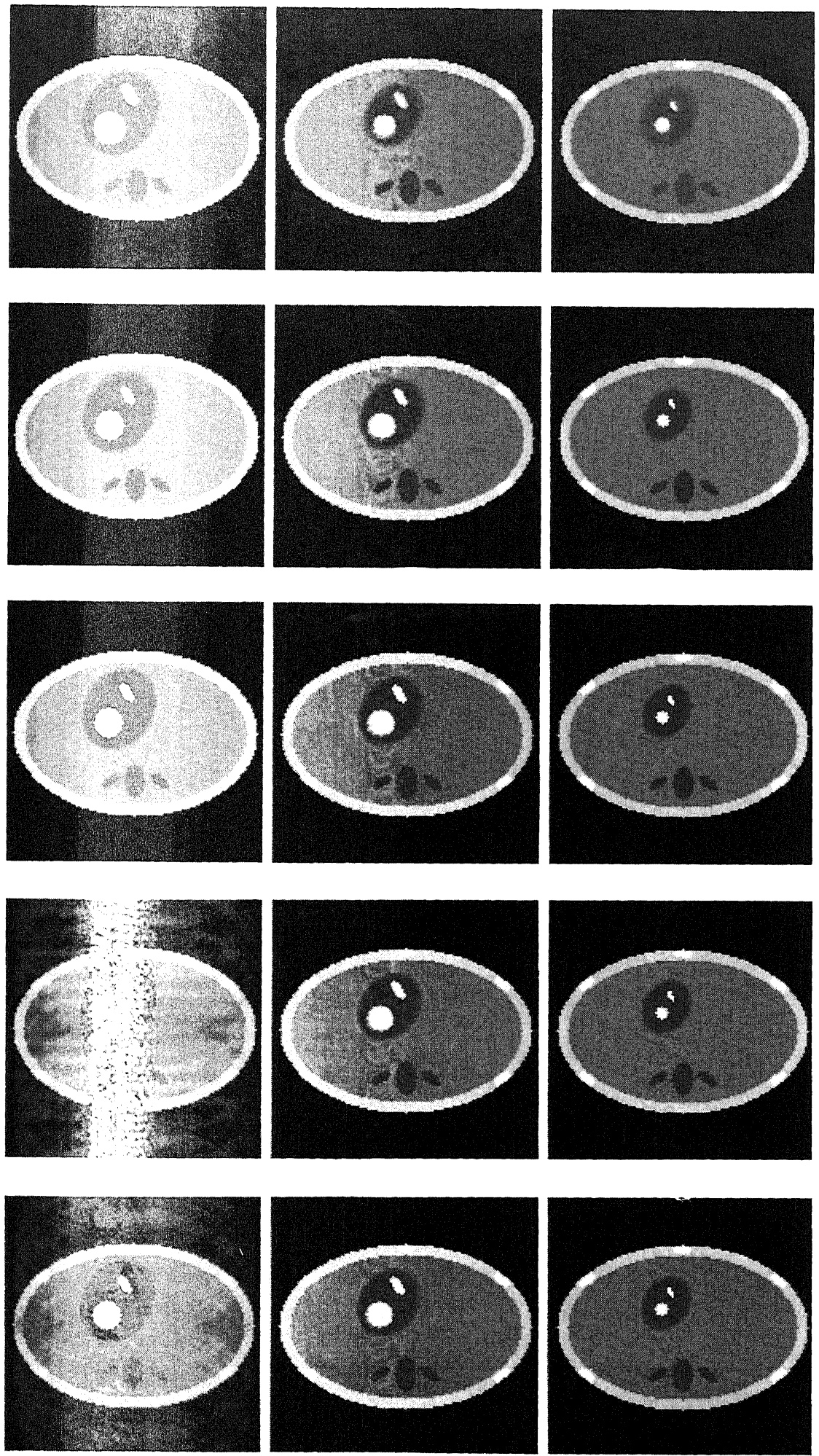

Figure 7. Reconstructions at phases 0,3 and 6 , from left to right. $\left(N_{\mathrm{pr}}=15\right)$. First row: order 0 ; second row: order 1; third row: order 3; fourth row: sinc; fifth row: reg. sinc. 

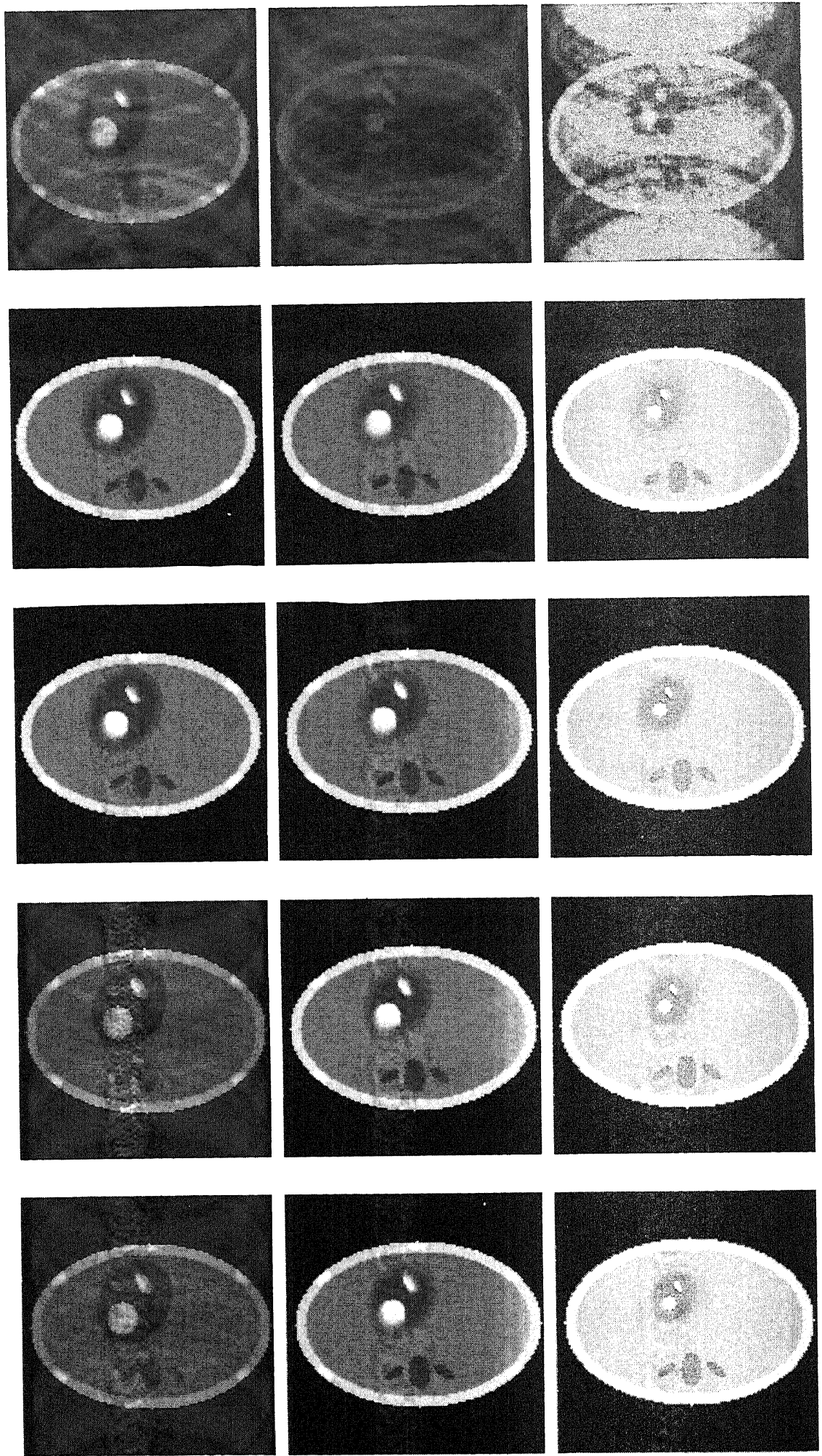

Figure 8. Reconstructions at phases 0,3 and 6 , from left to right. $\left(N_{\mathrm{p}, \mathrm{r}}=5\right)$. First row: order 0 ; second row: order 1; third row: order 3; fourth row: sinc; fifth row: reg. sinc. 
Table 2 The reconstruction error at eight phases for various interpolation algorithms, with $N_{\mathrm{pr}}=5$. Phase $\phi_{i}$ corresponds to time $i / \Phi_{\max }$, with $\Phi_{\max }=8$

\begin{tabular}{lcccccccc}
\hline & $\phi_{0}$ & $\phi_{1}$ & $\phi_{2}$ & $\phi_{3}$ & $\phi_{4}$ & $\phi_{5}$ & $\phi_{6}$ & $\phi_{7}$ \\
\hline Order 0 & 43.4 & 43.3 & 44.6 & 73.0 & 74.9 & 57.1 & 71.8 & 41.7 \\
Order 1 & 9.20 & 7.14 & 7.84 & 10.2 & 8.37 & 4.80 & 5.65 & 11.1 \\
Order 3 & 9.82 & 7.70 & 8.34 & 11.1 & 9.24 & 5.43 & 5.81 & 10.2 \\
Sinc & 43.3 & 24.7 & 10.9 & 13.7 & 10.5 & 8.71 & 8.08 & 15.9 \\
Reg. sinc & 39.8 & 18.4 & 9.92 & 11.7 & 10.2 & 6.75 & 7.64 & 14.7 \\
\hline \hline
\end{tabular}

Table 3 The reconstruction error at eight phases for various interpolation algorithms, with $N_{\mathrm{pr}}=5$. Phase $\phi_{i}$ corresponds to time $i / \Phi_{\max }$, with $\Phi_{\max }=8$

\begin{tabular}{lcccccccc}
\hline & $\phi_{0}$ & $\phi_{1}$ & $\phi_{2}$ & $\phi_{3}$ & $\phi_{4}$ & $\phi_{5}$ & $\phi_{6}$ & $\phi_{7}$ \\
\hline Order 0 & 9.67 & 3.31 & 11.5 & 11.3 & 7.13 & 2.29 & 8.35 & 11.8 \\
Order 1 & 5.33 & 2.15 & 4.57 & 6.48 & 4.69 & 1.69 & 2.78 & 6.42 \\
Order 3 & 5.65 & 2.71 & 5.01 & 6.80 & 4.80 & 2.10 & 3.01 & 6.74 \\
Sinc & 89.1 & 10.6 & 6.84 & 7.29 & 5.06 & 3.34 & 5.77 & 17.7 \\
Reg. sinc & 36.8 & 4.98 & 6.45 & 7.02 & 5.04 & 3.04 & 3.85 & 7.48 \\
\hline \hline
\end{tabular}

the interpolating function. When $N_{\mathrm{pr}}=15$, all reconstructions become comparable, except for the initial and final phases of the sinc reconstruction and, to a lesser extent, the reg. sinc reconstruction.

One sees in all reconstructions (in particular for $N_{\mathrm{pr}}=5$ ) a vertical band of noise superimposed on the region of the image which contains the moving ellipses. This artefact is a combined effect of the motion and the non-uniform sampling in time. That the band runs in the vertical rather than the horizontal direction is caused by the particular measuring strategy of retrospective gating: the main asynchronicity is in Fourier coefficients with different values of $k_{y}$, whereas Fourier coefficients with different values of $k_{x}$ are measured at virtually the same time. This artefact turns up in the reconstruction of real MR-images too.

To obtain an objective quality measure, we also compared the differences of the original and the reconstructions in $L^{2}$-norm, i.e. we computed

$$
\text { error : }=\sum_{x=0}^{x_{\max }} \sum_{y=0}^{-1}\left\{f_{\text {org }}(x, y, \text { phase })-f_{\text {rec }}(x, y, \text { phase })\right\}^{2},
$$

where $f_{\text {org }}$ and $f_{\text {rec }}$ denote the original and the reconstructed image respectively (see Tables 2 and 3$)$.

\subsection{Reconstruction from perturbed synthetic data}

In order to test how stable the algorithms are, we perturbed the measured Fourier coefficients (amplitude error) and the measurement times (time jitter error), respectively. 

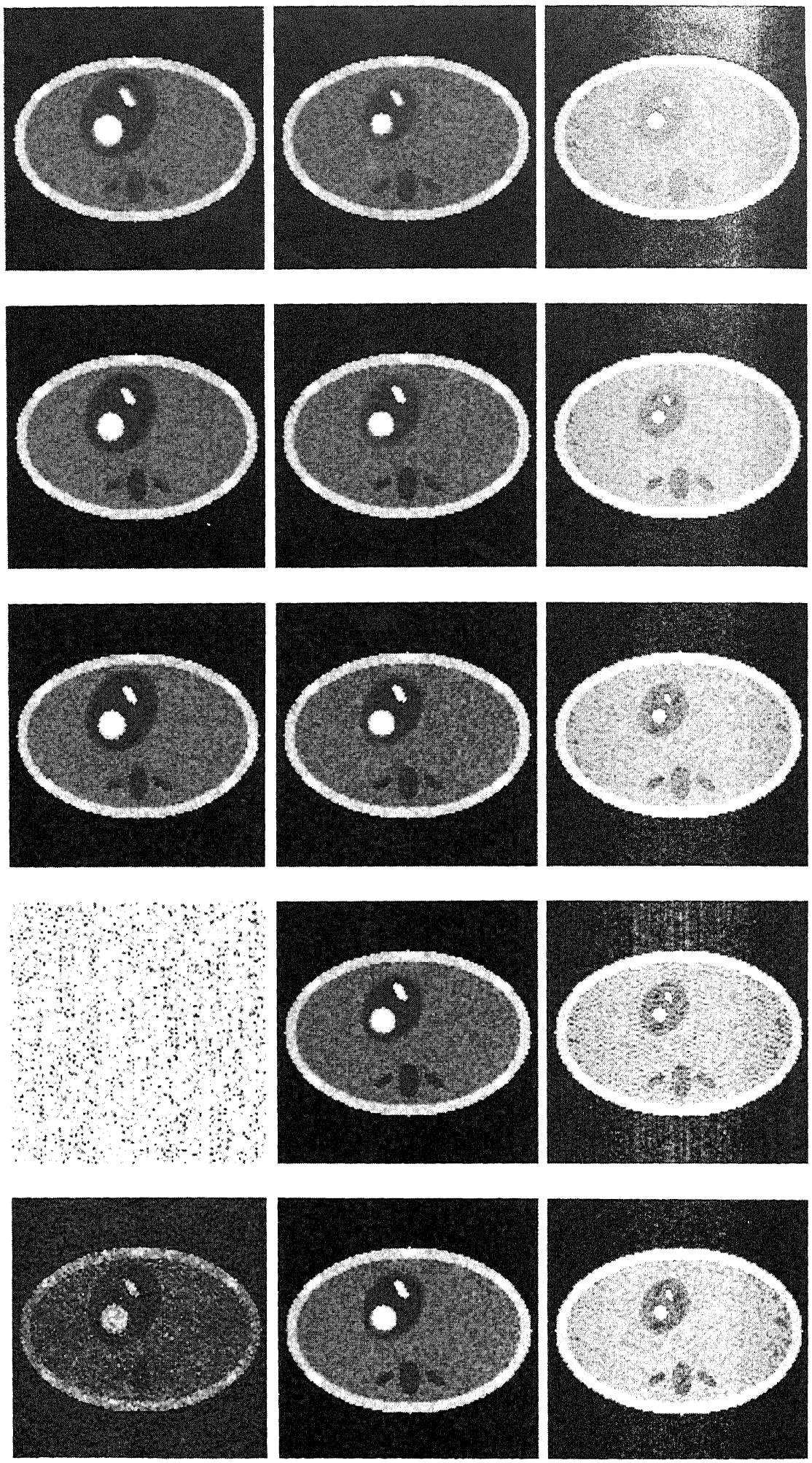

FIGURE 9. Reconstructions from perturbed data at phases 0,3 and 6 , from left to right. $\left(N_{\mathrm{pr}}=\right.$ 15). First row: order 0 ; second row: order 1 ; third row: order 3 ; fourth row: sinc; fifth row: reg. sinc. 
Table 4 Reconstruction error at eight phases from perturbed Fourier coefficients

$$
\left(N_{\mathrm{pr}}=15\right)
$$

\begin{tabular}{lcccccccc}
\hline \hline & $\phi_{0}$ & $\phi_{1}$ & $\phi_{2}$ & $\phi_{3}$ & $\phi_{4}$ & $\phi_{5}$ & $\phi_{6}$ & $\phi_{7}$ \\
\hline Order 0 & 20.0 & 18.0 & 21.2 & 21.1 & 19.4 & 17.7 & 19.6 & 21.5 \\
Order 1 & 19.2 & 18.3 & 18.6 & 19.7 & 19.0 & 18.3 & 18.5 & 19.2 \\
Order 3 & 22.7 & 22.8 & 23.1 & 23.2 & 22.6 & 23.0 & 22.4 & 23.6 \\
Sinc & 390 & 46.6 & 24.6 & 23.1 & 22.1 & 24.2 & 34.4 & 79.7 \\
Reg. sinc & 53.4 & 24.7 & 23.3 & 23.0 & 22.7 & 22.5 & 22.7 & 24.7 \\
\hline \hline
\end{tabular}

\subsubsection{Reconstructions from perturbed Fourier coefficients}

We added a random number $\eta_{k, i}$ to the data $g_{k, i}$ (for all $k \in \mathbb{K}$, and $i \in \mathbb{Q}$ ), i.e. the perturbed data become

$$
g_{k, i}^{\prime}:=g_{k, i}+\eta_{k, i}, \quad k \in \mathbb{K}, i \in \mathbb{Q} .
$$

The (complex) random numbers $\eta_{k, i}$ are chosen independently such that their real and imaginary parts are uniformly distributed in the interval $[-\sigma, \sigma]$. The different types of reconstructions corresponding to the perturbed data are shown in Fig. 9 where the value of $\sigma$ is chosen so large $(\sigma=4000)$ that a marked effect on the reconstructions becomes visible.

According to formula (3.20), the bound on the amplitude error for all reconstruction algorithms depends on the norm of the inverse of the Gram matrix and on the difference between $\left\{g_{k, i}\right\}$ and $\left\{g_{k, i}^{\prime}\right\}$ in $\ell^{2}$-norm. This is in agreement with what one may visually infer from the figures, which again show that in the case of sinc-reconstruction the problem is severely ill-conditioned, which is much improved by regularization. Table 4 gives the difference between the reconstruction and the chest phantom in $L^{2}$-norm. Note the complete degradation of the sinc-reconstruction at phase 0 . The other reconstructions now essentially have the same quality.

\subsubsection{Reconstructions from perturbed measurement times}

Finally, we discuss the effect due to perturbation of the measurement times. We added a real random number $\eta_{k, i}$ to the measurement time $t_{k, i}$ (for all $k \in \mathbb{K}$, and $i \in \mathbb{Q}$ ), i.e. the perturbed measurement times become

$$
t_{k, i}^{\prime}:=t_{k, i}+\eta_{k, i}, \quad k \in \mathbb{K}, i \in \mathbb{U} .
$$

The random numbers $\eta_{k, i}$ are chosen uniformly and independently from the interval $[-0.08,0.08]$. The reconstructions corresponding to the perturbed measurement times are shown in Fig. 10.

From this figure and the error results in Table 5 (particularly at phase 0 ) we observe that the sinc, order 1 and order 3 reconstruction do not behave well for the time jitter error. This is in agreement with formula (3.26), which shows that the bound on the aliasing error depends on the time derivative of the interpolating function. We also observe the 

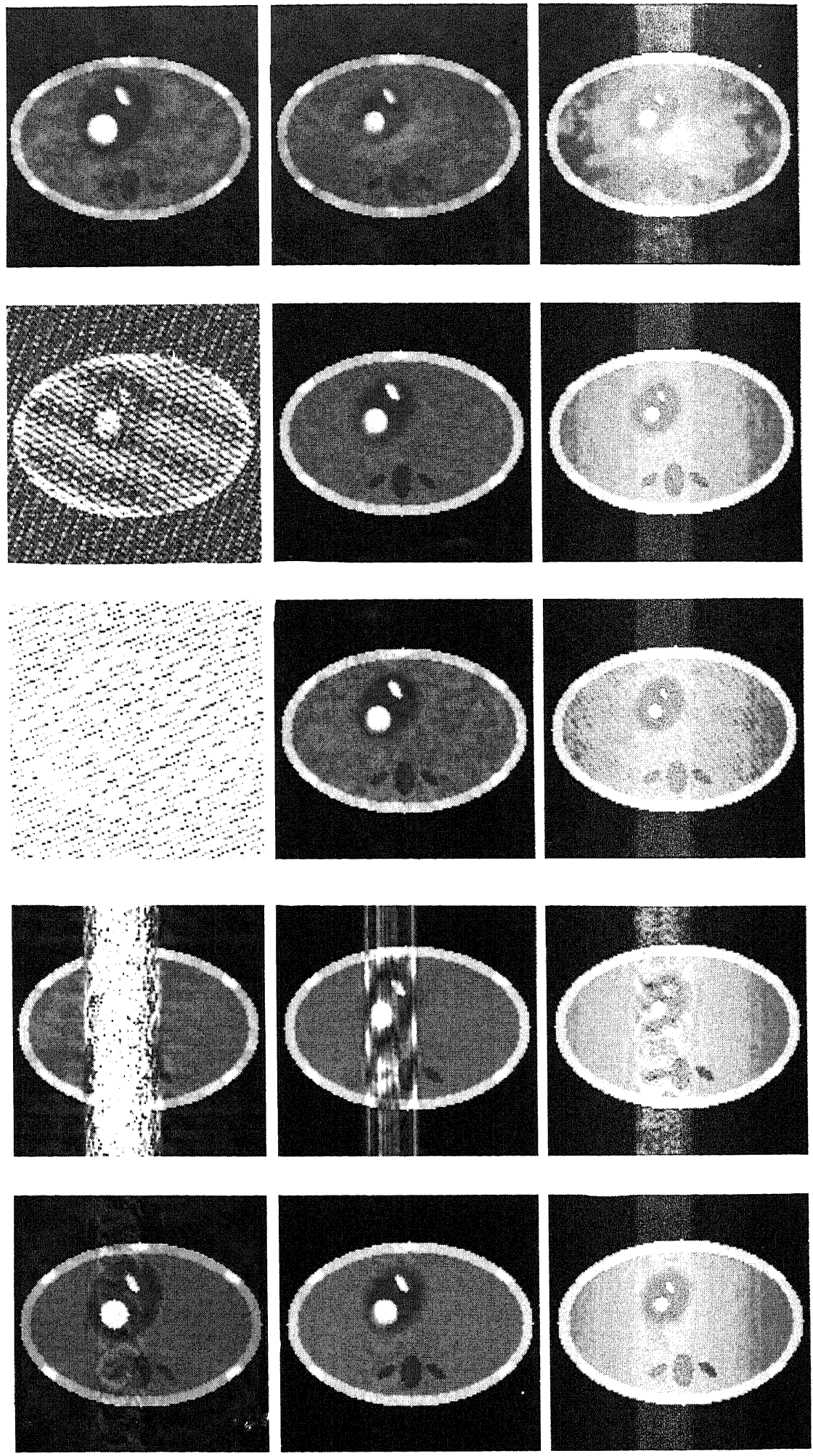

FigURE 10. Reconstructions from perturbed time markers at phases 0,3 and 6 , from left to right. ( $N_{\mathrm{pr}}$ $=15$ ). First row: order 0 ; second row: order 1 ; third row: order 3; fourth row: sinc; fifth row: reg. sinc. 
Table 5 Reconstruction error at eight phases from perturbed time markers $\left(N_{\mathrm{pr}}=15\right)$

\begin{tabular}{lcccccccc}
\hline & $\phi_{0}$ & $\phi_{1}$ & $\phi_{2}$ & $\phi_{3}$ & $\phi_{4}$ & $\phi_{5}$ & $\phi_{6}$ & $\phi_{7}$ \\
\hline Order 0 & 16.6 & 17.9 & 33.7 & 20.9 & 12.5 & 17.2 & 18.2 & 12.8 \\
Order 1 & 108 & 5.15 & 6.79 & 9.28 & 7.22 & 3.77 & 4.66 & 8.63 \\
Order 3 & 417 & 96.5 & 13.8 & 13.5 & 10.9 & 6.68 & 13.4 & 318 \\
Sinc & 207 & 38.7 & 63.0 & 35.4 & 9.75 & 8.96 & 16.7 & 69.9 \\
Reg. sinc & 26.0 & 5.46 & 6.79 & 8.48 & 7.07 & 3.67 & 5.01 & 8.46 \\
\hline \hline
\end{tabular}

appearance of small white 'clouds' in order 0-3 reconstruction. The sinc-reconstruction is again very bad, but (except for the initial phase) the regularized sinc-reconstruction is now the best of all reconstruction algorithms.

\section{Reconstruction of real MR-images}

In this section we present reconstructions from real MRI data. We obtained the time points $t_{k, i}$ by means of linear stretching, formula (2.11). The parameters in the reconstructions are: $k_{x}^{\max }=k_{y}^{\max }=128, N_{\mathrm{pr}}=50$. Results, at phase 4 of the heart cycle, are shown in Fig. 11 . A sketch of the anatomical details is given in Fig. 12. The two ellipse-like structures on the left and the right side are cross-sections of the arms. The big circular part is the bone of the chest. The heart is located at the front side of the chest (in the picture it is at the top). The grey part of the heart is the muscle tissue and the lighter parts are the heart-chambers.

Interesting features which can be deduced from MR-images, especially in movie mode, are (i) passive movement of the heart-muscle, (ii) a black dot can on the muscle tissue in the case of a heart attack, (iii) the ventricular, or atrial septal defect (this is a small hole in the tissue that separates the ventricles or the atria respectively). Important in this case is how well the heart muscle (in particular, its boundary) is reconstructed, and how little noise is contained in those parts of the image which contain moving heart structures. In the order 0 reconstruction the contours of the heart muscle are somewhat vague. In the order 1 and order 3 reconstruction they are less vague with lesser noise on the image than the order 0 reconstruction. The regularized sinc-reconstruction behaves better than sinc reconstruction, but is not a real improvement compared to order 0 reconstruction.

\section{Discussion and future prospects}

The main goal of this paper has been to perform a quantitative comparison of various reconstruction algorithms for cardiac magnetic resonance imaging which all attempt data synchronization after completion of the data acquisition process. We formulated a mathematical model describing the measurement process and solved the model equations in a Hilbert space framework. It turns out that the solution consists of two parts: (i) perform an interpolation in time for each set of spatial Fourier coefficients $\hat{f}\left(k_{x}, k_{y}, t_{k, i}\right)_{i \in 0}$; (ii) do the inverse Fourier transform to obtain $f\left(r, \phi_{i}\right)$ for a number of fixed phases $\phi_{i}=$ $i / \Phi_{\max }, i=0,1, \ldots, \Phi_{\max }-1$. We considered various interpolation methods: piecewise 

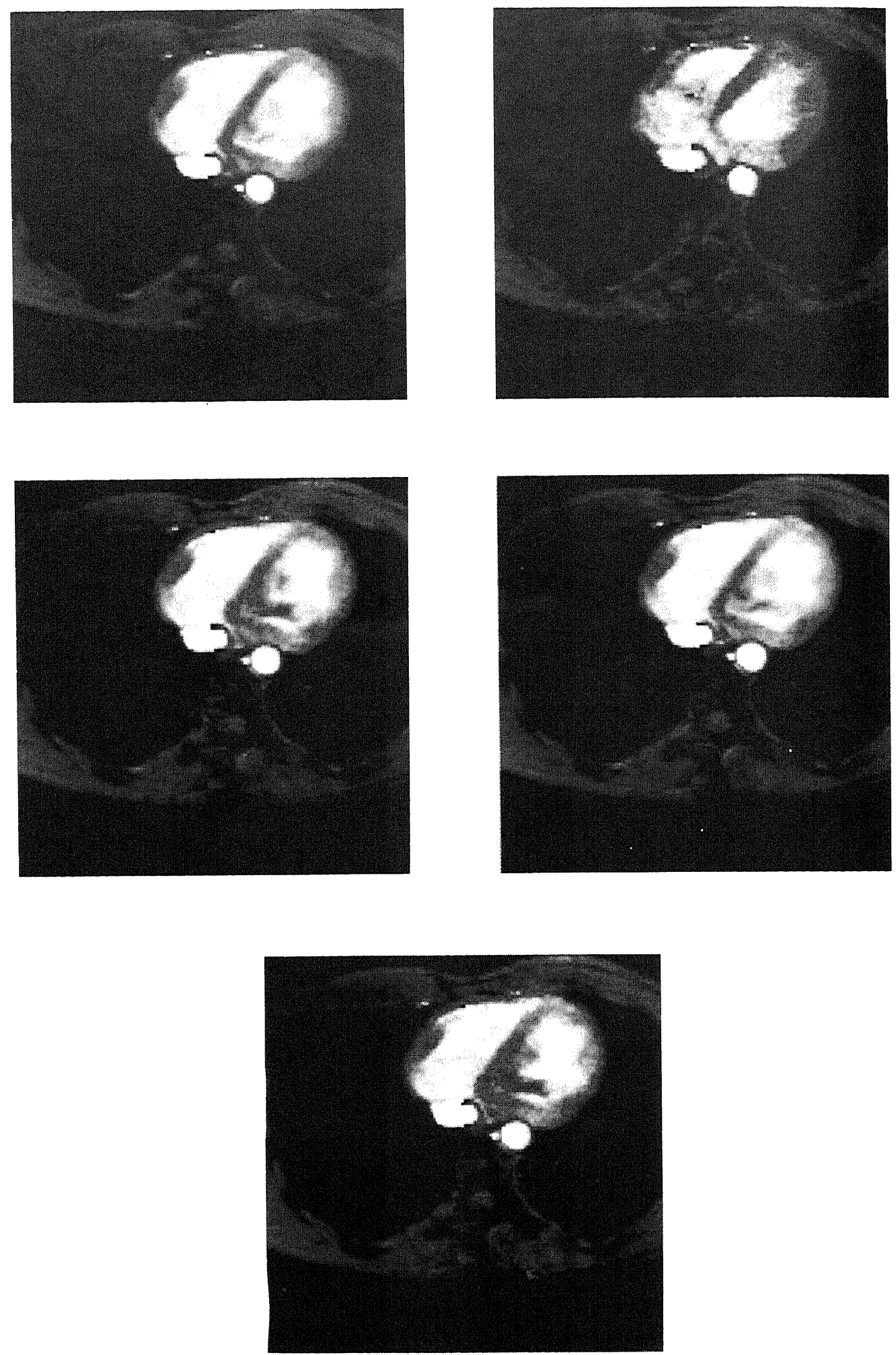

FIGURE 11. Reconstructions of MR images at phase 4. Top left: order 0 ; Top right: order 1; Middle left: order 3; Middle right: sinc; Bottom: reg. sinc. 
right ventricle

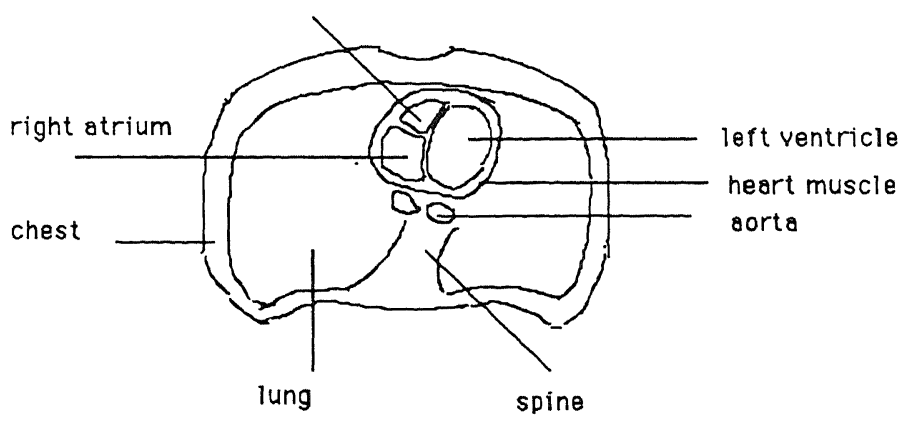

RIGHT

LEFT

FIGURE 12. Sketch of anatomic details of the cross-section of the chest.

constant ('order 0 '), which is the method originally used by Bohning [14]; piecewise linear ('order 1'); cubic spline ('order 3'); sinc-reconstruction ('sinc'); and the Tichonov-Phillips regularization of the sinc-reconstruction ('reg. sinc'). The algorithms were tested on synthetic as well as real data. In the case of real data it is unavoidable that there are model deficiencies, since many (physical) effects are not correctly incorporated in the model. For example, we completely neglect relaxation times and assume that what is measured is exactly the spatial Fourier transform of the proton density. More importantly, we have made the basic assumption that the beating heart behaves essentially the same in all heart beats, apart from a linear rescaling of the time points to a standard heart interval.

Therefore we have developed a chest phantom, i.e. a sequence of synthetic images consisting of a number of moving ellipses, for which we imitated the measuring process as well as the precise form of rescaling. The goal of these simulations is to test the accuracy of the temporal synchronization process, leaving out all other factors which complicate the reconstruction in practice. Quantitative comparisons have been made of the various interpolation routines by computing the difference in $L^{2}$-norm between the original and the reconstruction, which show that for unperturbed data the order 1 and order 3 reconstructions are best. The differences between the various methods increase as the average number $N_{\mathrm{pr}}$ of profiles is decreased. The sinc-reconstruction suffers from the defect that, in contrast to the case of order 1 and order 3 interpolation, one cannot incorporate periodicity of the interpolating function, which causes the first and last phases of the reconstruction to be poorer in quality than the middle phases. This could be improved by periodically extending the data points and perform the sinc-reconstruction on the enlarged data set. A disadvantage would be that the corresponding Gram matrices increase in size. We also did some tests with perturbed Fourier coefficients and measurement times to determine stability under perturbation. It turns out that in that case the regularized sincreconstruction is the most robust method. Cubic spline interpolation is very sensitive to perturbation of the measurement times.

For the case of real data we find from visual inspection of the images that order 1 and order 3 behave equally well and better than order 0 , while (regularized) sinc gives inferior results. The comparison with the reconstructions from synthetic data is complicated by the 
fact that in this case the number of profiles is fairly large $\left(N_{\mathrm{pr}}=50\right)$. However, since order 1 and order 3 reconstruction are robust and fast they should be preferred to order 0 in practical applications.

A final remark concerns the so-called 'breathing artifact', which is caused by respiratory motion and produces ghosts of the moving parts in the image [2]. The phenomenon can be explained as a kind of aliasing or interference effect between the periodicity of the respiratory motion and the periodic increase of the magnetic field gradient in the phaseencoding direction. The effect is most prominent if the patient during data acquisition is breathing in a very regular way. For the data set used in this paper this was apparently not the case. A method, called Respiratory Ordered Phase Encoding (ROPE), has been proposed to correct for the breathing artifact [24]. Here one needs information about the respiratory motion during the data acquisition, so in this sense the method is based on the same principle as ordinary cardiac gating. An alternative which suggests itself in the light of the present paper, is to develop a 'retrospective cardiac-respiratory gating method', in which one would register both the electrocardiogram and the respiratory motion during scanning, and order the data retrospectively both with respect to the cardiac as well as to the respiratory state. A crucial question which would have to be answered is whether accurate monitoring of the respiratory motion is feasible.

\section{Acknowledgement}

We thank P. Hofstee and B. Lisser for extensive programming assistance. One of us (M.Z.) gratefully thanks Prof. Dr. G. Y. Nieuwland for his stimulating ideas and his enthusiastic advice. Finally, we are indebted to Dr. Ir. F. A. Kuipers and Ir. M. Fuderer of the Philips Medical Systems Division (Best, The Netherlands) for making their MRI data available and giving practical advice whenever needed.

\section{Appendix: List of symbols}

$\begin{array}{ll}\omega_{L} & \text { Larmor frequency } \\ \omega_{r f} & \text { frequency of RF pulse } \\ m_{\perp} & \text { transverse magnetization } \\ m_{z} & \text { longitudinal magnetization } \\ \delta t & \text { time between two samples in a profile } \\ \Delta G_{y} & \text { step size of the phase-encoding gradient } \\ L_{x} & \text { field of view in the } x \text {-direction } \\ L_{y} & \text { field of view in the } y \text {-direction } \\ \boldsymbol{r} & \text { position vector }(x, y) \\ X_{\max } & \text { number of horizontal pixels in the image plane } \\ Y_{\max } & \text { number of vertical pixels in the image plane } \\ \xi & \text { spatial Fourier variable } \\ \omega & \text { temporal Fourier variable } \\ G_{x} & \text { readout gradient } \\ G_{y} & \text { phase-encoding gradient } \\ G_{z} & \text { slice selection gradient }\end{array}$


$\Delta \xi_{x} \quad$ horizontal sampling distance in the spatial Fourier plane $\left(=2 \pi / L_{x}\right)$

$\Delta \xi_{y} \quad$ vertical sampling distance in the spatial Fourier plane $\left(=2 \pi / L_{y}\right)$

$k_{x} \quad$ integer counting the number of horizontal samples in the spatial Fourier plane

$k_{y} \quad$ integer counting the number of vertical samples in the spatial Fourier plane

$k_{x}^{\max } \quad$ maximum value of $k_{x}$

$k_{y}^{\max } \quad$ maximum value of $k_{y}$

$\boldsymbol{k} \quad$ wave vector $\left(k_{x}, k_{y}\right)$

$i \quad$ integer counting the number of profiles for a fixed value of $k_{y}$

$R_{k} \quad$ time of the $k$ th $\mathrm{R}$-wave

$N_{\mathrm{pr}} \quad$ total number of profiles for a fixed value of $k_{y}$

$T_{\text {rep }} \quad$ repetition time between profiles

$T_{\text {acq }} \quad$ measurement time of a profile

$\bar{T}_{\mathrm{RR}} \quad$ average duration of a heartbeat

$\Phi_{\max }$ integer counting the number of phases to be reconstructed

$\epsilon \quad$ relative variation of duration of heartbeats

$\tau \quad$ actual time during acquisition $(-\infty<\tau<\infty)$

$t \quad$ rescaled time or relative heart phase $(\tau \in[0,1])$

$f(\boldsymbol{r}, t)$ proton density at position $\boldsymbol{r}$ and heart phase $t$

$\hat{f}(\boldsymbol{k}, t)$ spatial Fourier coefficient of the proton density $f$ with wave vector $\boldsymbol{k}$ at heart phase $t$

$f(\boldsymbol{r}) \quad$ the function $t \mapsto f(\boldsymbol{r}, t)$

$\hat{f}(\boldsymbol{k}) \quad$ the function $t \mapsto \hat{f}(\boldsymbol{k}, t)$

$\gamma \quad$ regularization parameter

\section{References}

[1] MCKinnon, G. C. \& Bates, R. H. T. 1981 Towards imaging the beating heart usefully with a conventional CT scanner. IEEE Trans. Biomed. Engineering 28, 123-127.

[2] Lenz, G. W., HaAcke, E. M. \& White, R. D. 1989 Retrospective cardiac gating: a review of technical aspects and future directions. Magn. Res. Imag. 7, 445-455.

[3] Hinshaw, W. S. \& Lent, A. H. 1983 An introduction to NMR imaging: from the Bloch equation to the imaging equation. Proc. IEEE 71, 338-350.

[4] KING, K. F. \& MoRAN, P. R. 1984 A unified description of NMR imaging, data-collection strategies, and reconstruction. Med. Phys. 11, 1-14.

[5] Mansfield, P. \& Morris, P. G. 1982 NMR Imaging in Biomedicine. Academic Press.

[6] TwIEG, D. B. 1983 The $k$-trajectory formulation of the NMR imaging process with applications in analysis and synthesis of imaging methods. Med. Phys. 10, 610-621.

[7] ZwaAn, M. 1989 Dynamic MRI reconstruction. (To appear in Math. Meth. Appl. Sc., cf. CWIReports AM-R8905, AM-R8907, CWI, Amsterdam.)

[8] ZWAan, M. 1990 Approximation of the solution to the moment problem in a Hilbert space. Numer. Funct. Anal. Opt. 11, 601-608.

[9] ZwaAn, M. 1990 Dynamic MRI reconstruction as a moment problem. Part III. An error analysis of reconstruction by sinc and spline interpolation in a Hilbert space setting. CWIReport AM-R9002, CWI, Amsterdam.

[10] Nussbaumer, H. J. 1982 Fast Fourier Transform and Convolution Algorithms. Springer-Verlag.

[11] HARRIS, F. J. 1978 On the use of windows for harmonic analysis with the discrete Fourier transform. Proc. IEEE 66, 51-83. 
[12] VAN Disk, P. 1984 ECG-triggered NMR imaging of the heart. Diag. Imag. Clin. Med. 53, 29-37.

[13] Glover, G. H. \& Pelc, N. J. 1988 A rapid-gated cine MRI technique. In: Magnetic Resonance Annual 1985, pp. 299-333. Raven Press.

[14] Bohning, D. E. 1988 Cardiac Gating Strategies. In: New Concepts in Cardiac Imaging. Year Book Medical Publishers.

[15] ZWAAN, M. 1990 Error estimates for nonuniform sampling. Numer. Funct. Anal. Opt. 11, 589-599.

[16] Bertero, M., De Mol, C. \& Pike, E. R. 1985 Linear inverse problems with discrete data. I. General formulation and singular system analysis. Inverse Problems 1, 301-330.

[17] Greville, T. N. E. 1969 Theory and Applications of Spline Functions. Academic Press.

[18] Bertero, M., De Mol, C. \& Pike, E. R. 1988 Linear inverse problems with discrete data. II. Stability and regularisation. Inverse Problems 4, 573-594.

[19] Natterer, F. 1986 The Mathematics of Computerized Tomography. B. G. Teubner \& John Wiley.

[20] Beutler, F. J. \& Root, W. L. 1976 The operator pseudoinverse in control and systems identification. In Generalized Inverses and Applications (ed. M. Z. Nashed). Academic Press.

[21] Lewis, T. O. \& Odell, P. L. 1971 Estimation in Linear Models. Prentice-Hall.

[22] Ogawa, H. \& OJa, E. 1986 Projection filter, Wiener filter, and Karhunen-Loève subspaces in digital image restoration. J. Math. Anal. Appl. 114, 37-51.

[23] Louis, A. K. 1989 Inverse und schlecht gestellte Probleme. B. G. Teubner, Stuttgart.

[24] Bailes, D. R., Gilderdale, D. J., Bydder, G. M., Collins, A. G. \& Firmin, D. N. 1985 Respiratory ordered phase encoding (ROPE). A method for reducing respiratory motion artefacts in MR Imaging. J. Computer Assisted Tomography 9, 835-838. 